\title{
Are Striatal Tyrosine Hydroxylase Interneurons Dopaminergic?
}

\author{
๑Harry S. Xenias, Osvaldo Ibáñez-Sandoval, Tibor Koós, and ๑James M. Tepper \\ Center for Molecular and Behavioral Neuroscience, Rutgers University, Newark, New Jersey 07102
}

Striatal GABAergic interneurons that express the gene for tyrosine hydroxylase $(\mathrm{TH})$ have been identified previously by several methods. Although generally assumed to be dopaminergic, possibly serving as a compensatory source of dopamine (DA) in Parkinson's disease, this assumption has never been tested directly. In TH-Cre mice whose nigrostriatal pathway had been eliminated unilaterally with 6-hydroxydopamine, we injected a Cre-dependent virus coding for channelrhodopsin-2 and enhanced yellow fluorescent protein unilaterally into the unlesioned midbrain or bilaterally into the striatum. Fast-scan cyclic voltammetry in striatal slices revealed that both optical and electrical stimulation readily elicited DA release in control striata but not from contralateral striata when nigrostriatal neurons were transduced. In contrast, neither optical nor electrical stimulation could elicit striatal DA release in either the control or lesioned striata when the virus was injected directly into the striatum transducing only striatal TH interneurons. This demonstrates that striatal TH interneurons do not release DA. Fluorescence immunocytochemistry in enhanced green fluorescent protein (EGFP)-TH mice revealed colocalization of DA, L-amino acid decarboxylase, the DA transporter, and vesicular monoamine transporter-2 with EGFP in midbrain dopaminergic neurons but not in any of the striatal EGFP-TH interneurons. Optogenetic activation of striatal EGFP-TH interneurons produced strong GABAergic inhibition in all spiny neurons tested. These results indicate that striatal TH interneurons are not dopaminergic but rather are a type of GABAergic interneuron that expresses TH but none of the other enzymes or transporters necessary to operate as dopaminergic neurons and exert widespread GABAergic inhibition onto direct and indirect spiny neurons.

Key words: dopamine; GABA; interneuron; neostriatum; optogenetics; voltammetry

\section{Introduction}

The neostriatum is the largest nucleus of the basal ganglia and receives the vast majority of its input. The principal striatal neuron is the spiny projection neuron (SPN), which accounts for $\sim 95 \%$ of its neuronal population (Mehler, 1981; Parent, 1986; Gerfen and Wilson, 1996). The remaining striatal neurons comprise a diverse group of interneurons (Kawaguchi, 1993; Tepper and Bolam, 2004; Tepper et al., 2010). Although far fewer in number than the SPNs, striatal interneurons are powerful determinants of striatal output (Koós and Tepper, 1999, 2002; Wilson, 2007; Gittis et al., 2010; Ibáñez-Sandoval et al., 2010, 2011; Tepper et al., 2010; Klaus et al., 2011). Classically, striatal interneurons were considered to consist of four subtypes, a cholinergic interneuron and three types of GABAergic interneuron: (1) a parvalbumin-expressing fast-spiking interneuron; (2) a neuro-

Received Jan. 12, 2015; revised March 3, 2015; accepted March 15, 2015.

Author contributions: H.S.X., T.K., and J.M.T. designed research; H.S.X. and 0.I.-S. performed research; T.K. contributed unpublished reagents/analytic tools; H.S.X., 0.I.-S., and J.M.T. analyzed data; H.S.X. and J.M.T. wrote the paper.

This research was supported by National Institute of Neurological Disorders and Stroke Grants NS034865 (J.M.T.) and NS072950 (T.K. and J.M.T.) and Rutgers, The State University of New Jersey. We thank Fulva Shah, Arpan Garg, Parth Gandhi, Janish Kothari and Leticia Maldonado for their technical assistance.

The authors declare no competing financial interests.

Correspondence should be addressed to Dr. J. M. Tepper, Center for Molecular and Behavioral Neuroscience, Rutgers, The State University of New Jersey, 197 University Avenue, Newark, NJ 07102. E-mail: jtepper@andromeda.rutgers.edu.

DOI:10.1523/JNEUROSCI.0195-15.2015

Copyright $\odot 2015$ the authors $\quad 0270-6474 / 15 / 356584-16 \$ 15.00 / 0$ peptide Y/somatostatin/nitric oxide synthase plateau lowthreshold spiking interneuron; and (3) a calretinin interneuron (Tepper and Bolam, 2004). The electrophysiological properties of the first three of these were first described in a landmark in vitro recording study by Kawaguchi (1993). The physiological properties of the calretinin interneuron remained unknown.

Subsequently, experiments in mice that expressed enhanced green fluorescent protein (EGFP) under the control of the tyrosine hydroxylase (TH) promoter (Gong et al., 2003) revealed the existence of a substantial population of EGFP-TH interneurons (Ibáñez-Sandoval et al., 2010). This was not the first description of the existence of striatal TH neurons, but it provided the first direct evidence that they were interneurons, received cortical and striatal inputs, and exerted powerful GABAergic inhibition onto SPNs.

Striatal TH interneurons (THINs) were first described immunocytochemically in the primate (Dubach et al., 1987) and subsequently in a host of other species, including mice (Mao et al., 2001; Petroske et al., 2001), rats (Meredith et al., 1999; Robinson et al., 2002), and humans (Porritt et al., 2000; Petroske et al., 2001; Cossette et al., 2004, 2005a,b). Interest in these neurons intensified after it was reported consistently that the number of striatal THINs increased greatly after 6-hydroxydopamine (6OHDA) or MPTP lesions that destroyed the nigrostriatal dopamine (DA) innervation. Many assumed that the striatal THINs were dopaminergic and might therefore serve as an intrinsic source of DA that could compensate for the loss of DA in exper- 
imental or idiopathic Parkinson's disease (PD; Betarbet et al., 1997; Meredith et al., 1999; Porritt et al., 2000; Robinson et al., 2002; Cossette et al., 2005a; Tandé et al., 2006; Huot and Parent, 2007; Darmopil et al., 2008; Gittis and Kreitzer, 2012).

However, all of the evidence that striatal THINs are dopaminergic is indirect and comes exclusively from immunocytochemical studies, most of which did not attempt to determine the colocalization of DA and TH. There is no direct evidence that striatal THINs either express DA or release DA during stimulation. In the present studies, we used ex vivo voltammetry and optogenetics in $\mathrm{TH}-\mathrm{Cre}$ mice to determine whether selective optical activation of striatal THINs transduced with channelrhodopsin2 (ChR2) could elicit striatal DA release and EGFP-TH mice and immunocytochemistry to determine whether striatal THINs express DA, aromatic L-amino acid decarboxylase (AADC), vesicular monoamine transporter-2 (VMAT2), and/or the DA transporter (DAT).

\section{Materials and Methods}

Subjects. Striatal THINs were studied in a mouse BAC transgenic THCre [ $\mathrm{Tg}(\mathrm{TH}-\mathrm{Cre}) 12 \mathrm{Gsat}$; Gene Expression Nervous System Atlas (GENSAT); nine males and six females] and another BAC transgenic mouse expressing EGFP under the control of the TH promotor [Tg(Th-EGFP) 1Gsat/MNmnc], simply referred hereafter as TH-Cre and EGFP-TH mice (three males and two females), respectively. EGF$\mathrm{P}-\mathrm{TH}$ mice were acquired initially from the Mutant Mouse Regional Resource Center at University of California, Los Angeles, derived from GENSAT (http://www.mmrrc.org/strains/292/0292.html). Mice were bred at our Association for Assessment and Accreditation of Laboratory Animal Care-accredited animal colony and genotyped from tail samples. All surgical procedures were performed in accordance with the National Institutes of Health Guide to the Care and Use of Laboratory Animals and with the approval of the Rutgers University Institutional Animal Care and Use Committee.

Viral-mediated gene transfer. We used Cre-based optogenetic methods established previously in our laboratory (Tecuapetla et al., 2010; English et al., 2012). Briefly, the fusion gene encoding ChR2 and enhanced yellow fluorescent protein (EYFP) was delivered via a Cre-dependent adenoassociated virus [AAV5-EF1a-DIO-hChR2(H134R)-EYFP; University of North Carolina, Vector Core Services, Chapel Hill, NC], from here on simply referred to as AAV-ChR2-EYFP. A pair of incompatible loxP recombination sites oriented in an inverted open reading frame flanks the ChR2-EYFP fusion gene. During Cre recombination, the fusion gene is properly oriented and translated into ChR2-EYFP. When this viral construct is injected into the striatum or midbrain of TH-Cre mice, recombination results in the selective expression of ChR2-EYFP in cells that normally express the $\mathrm{TH}$ gene.

Intracerebral viral and 6-OHDA injections. Mice were anesthetized with isoflurane and affixed to a stereotaxic frame. After subcutaneous administration of bupivicaine, the scalp was reflected. Small burr holes were drilled bilaterally over the striatum [anteroposterior (AP), $0.5 \mathrm{~mm}$ from bregma; lateral (L), $1.85 \mathrm{~mm}$ from midline] for viral injections and over the midbrain (AP, $-3.3 \mathrm{~mm}$ from bregma; L, $0.8 \mathrm{~mm}$ from the midline) for the injection of 6-OHDA. Injections were done within a Biosafety Level-2 (BSL-2) rated isolation cabinet using a Nanoject II Auto-Nanoliter Injector (catalog \#3-000-204; Drummond Scientific Company) mounted on an MP-285 micromanipulator (Sutter Instruments). Glass capillaries ( $1.14 \mathrm{~mm}$ outer diameter, $0.53 \mathrm{~mm}$ inner diameter; catalog \#3-000-203-G/X; Drummond Scientific Company) were pulled on a Narishige PE-2 glass microelectrode puller (Narishige International). Pipette tips were cut back to an outer diameter of $\sim 40-50$ $\mathrm{mm}$. Virus $(0.83 \mu \mathrm{l})$ was injected bilaterally at two different dorsoventral coordinates to maximize the infected area at $23 \mathrm{nl} / \mathrm{s}$ at $-2.25 \mathrm{~mm}(0.5 \mu \mathrm{l})$ and $-3.0 \mathrm{~mm}(0.33 \mu \mathrm{l})$ from the surface of the brain. 6-OHDA was prepared in $0.9 \% \mathrm{NaCl}$ and $0.2 \%$ ascorbic acid at a concentration of 3.6 $\mathrm{mg} / \mathrm{ml}$. 6-OHDA $(0.8 \mu \mathrm{l})$ was injected into the midbrain unilaterally at a depth of $4.3 \mathrm{~mm}$ from the cortical surface. After completion of each injection, the pipette was kept in place for 10 min to prevent spread back along the pipette track and then gradually withdrawn. Injected animals were housed for a 7-10 d period under BSL-2 quarantine and experiments performed $14-21 \mathrm{~d}$ later.

Preparation of brain slices. Mice were overdosed with $150 \mathrm{mg} / \mathrm{kg}$ ketamine and $30 \mathrm{mg} / \mathrm{kg}$ xylazine intraperitoneally and perfused transcardially with an ice-cold modified Ringer's solution containing the following (in mM): 248 sucrose, $2.5 \mathrm{KCl}, 7 \mathrm{MgCl}_{2}, 23 \mathrm{NaHCO}_{3}, 1.2 \mathrm{NaH}_{2} \mathrm{PO}_{4}, 7$ glucose, 1 ascorbic acid, and 3 sodium pyruvate. The solution was bubbled before use with $95 \% \mathrm{O}_{2}$ and $5 \% \mathrm{CO}_{2}$. Brains were removed quickly, blocked, and cut into $300 \mu \mathrm{m}$ coronal or parahorizontal slices on a Vibratome 3000 . Slices were transferred immediately into a holding chamber containing normal Ringer's solution kept at $33-34^{\circ} \mathrm{C}$ and bubbled continuously with $95 \% \mathrm{O}_{2}$ and $5 \% \mathrm{CO}_{2}$, containing the following (in mm): $124 \mathrm{NaCl}, 2.5 \mathrm{KCl}, 1.2 \mathrm{NaH}_{2} \mathrm{PO}_{4}, 26 \mathrm{NaHCO}_{3}, 1.3 \mathrm{MgCl}_{2}, 2 \mathrm{CaCl}_{2}$, 10 glucose, 1 ascorbic acid, 3 pyruvate, and 0.4 myo-inositol. After a 5 min incubation period, slices were allowed to cool down to room temperature.

Fluorescent and bright-field imaging of slices. Slices were transferred into a recording chamber, perfused with the same modified Ringer's solution at $\sim 32-33^{\circ} \mathrm{C}$. Slices were visualized under either infrared or epifluorescence on a BX-50WI Olympus fixed-stage microscope with a digital frame transfer camera (Cooke SensiCam; PCO-Tech). ChR2EYFP-positive $\left(\mathrm{ChR} 2-\mathrm{EYFP}^{+}\right)$cells were first visualized under epifluorescence and patched in current clamp under infrared visualization using an oblique condenser, yielding a differential interference contrast-like image. Regions rich with fluorescent ChR2-EYFP ${ }^{+}$somata and/or processes were selected for voltammetric recordings, and the carbon fiber electrodes (CFEs) were positioned within these regions under visual guidance.

Ex vivo electrophysiology. Whole-cell recordings were obtained with a Multiclamp 700B amplifier (Molecular Devices) and an ITC-1600 digitizer (HEKA Elektronik). Data were acquired by a PowerMac G5 running Axograph (www.axographx.com) and digitized at $20 \mathrm{kHz}$. Micropipettes for patch-clamp recordings were made using thin-walled borosilicate pipettes with an outer diameter of $1.2 \mathrm{~mm}$ and inner diameter of 0.94 mm (catalog \#GC120T-7.5; Harvard Apparatus) and pulled on a Sutter P-97 micropipette puller (Sutter Instruments). Patch pipette resistances were typically $3 \mathrm{M} \Omega$. Whole-cell current-clamp recordings were performed using an internal solution containing the following: $130 \mathrm{~mm}$ K-gluconate, $10 \mathrm{~mm} \mathrm{NaCl}, 2$ mm $\mathrm{MgCl}_{2}, 10$ mм HEPES, 3 mм Na2-ATP, $0.3 \mathrm{~mm}$ GTP, $1 \mathrm{~mm}$ EGTA, $0.1 \mathrm{~mm} \mathrm{CaCl}_{2}$, and either $0.5 \%$ biocytin or 25 mu Alexa Fluor 594 (catalog \#A-10438; Invitrogen), pH 7.3-7.4.

Ex vivo voltammetry. Fast-scan cyclic voltammetry (FSCV; John and Jones, 2007) was performed with an Ensman EI-400 biopotentiostat (Cypress Systems). Scans were performed at $10 \mathrm{~Hz}$ over a potential range from -0.4 to $1.3 \mathrm{~V}$ (vs $\mathrm{Ag} / \mathrm{AgCl}$ ) at a rate of $400 \mathrm{~V} / \mathrm{s}$. Data were digitalized at 1000 points per scan and transferred to a personal computer via an NI PCI-6052E PC board (National Instruments). Data were analyzed and processed for graphical output by custom programs written by H.S.X. in MATLAB (MathWorks). CFEs were constructed from borosilicate glass capillaries (catalog \#GC200-10; Harvard Apparatus) and 7- to 8- $\mu \mathrm{m}$ diameter carbon fiber (Goodfellow) using standard techniques (Wiedemann et al., 1991; Millar and Pelling, 2001; Patel and Rice, 2006). Briefly, a CFE was fashioned by threading a carbon fiber into a glass capillary, which was then pulled on a Narishige PE-2 glass microelectrode puller (Narishige International), forming a tight glass seal around the carbon fiber. The exposed fiber was cut $30-70 \mu \mathrm{m}$ from the end of the glass seal with a scalpel mounted on a Narishige MX-1 micromanipulator (Narishige International). The internal carbon fiber was connected to a thin caliber wire with Woods metal (Goodfellow). DA was identified by its characteristic single oxidation and reduction peak potentials, at $\sim 0.7$ and $-0.3 \mathrm{~V}$ (Wiedemann et al., 1991), respectively. The CFE was positioned under visual control with an MP-225 micromanipulator (Sutter Instruments) $\sim 80 \mu \mathrm{m}$ beneath the surface of the slice. Electrical or optical stimuli were delivered every $30 \mathrm{~s}$ (see below for protocols). Extracellular concentrations of DA $\left([D A]_{\mathrm{e}}\right)$ were postcalibrated in the bath at $32-33^{\circ} \mathrm{C}$ with a $1 \mu \mathrm{M}$ DA standard for both control and drug-containing solutions. In addition, for some voltammetric experiments, L-DOPA-treated TH- 
Cre mice were used, following the same L-DOPA treatment protocol used for DA immunocytochemistry (see below).

Electrical stimulation. Local electrical stimulation consisted of single $250 \mu \mathrm{A}, 50 \mu$ s square-wave current pulses delivered through a concentric bipolar tungsten metal microelectrode (Harvard Apparatus), generated by an SC-100 constant-current stimulus isolation unit controlled through the digitizer and acquisition software. The stimulating microelectrode was positioned $100 \mu \mathrm{m}$ from the inserted tip of the CFE and at a depth of $\sim 50-80 \mu \mathrm{m}$ beneath the surface of the slice. Stimuli were delivered every $30 \mathrm{~s}$.

Optical stimulation. Neurons transduced with ChR2-EYFP were activated by single $2.5 \mathrm{~ms}$ pulses of blue light emitted by a $750 \mathrm{~mW}$ blue LED (Cree) positioned just below the condenser with the iris and sector stops fully opened. Timing was controlled through the digitizer by the data acquisition software. In some experiments, epifluorescence illumination was delivered by a xenon lamp and passed through a filter to deliver blue light of $470 \pm 20 \mathrm{~nm}$ wavelength (Chroma Technology). The duration of the epifluorescence stimulation was controlled with a Uniblitz shutter (Vincent Associates) by the acquisition software through the digitizer. Light pulses were delivered every $30 \mathrm{~s}$.

Biocytin histochemistry. Slices containing biocytin-filled neurons were transferred into a scintillation vial containing $\sim 2 \mathrm{ml}$ of $4 \%$ paraformaldehyde (PFA), microwave fixed to $\sim 60^{\circ} \mathrm{C}$ for $8-10 \mathrm{~s}$, and fixed overnight. Slices were then washed three times in $0.1 \mathrm{M}$ PBS and resectioned typically into three to four $60 \mu \mathrm{m}$ sections. After washing three times for $10 \mathrm{~min}$ in $0.1 \mathrm{M}$ PBS, sections were then washed in $10 \%$ methanol and $3 \%$ $\mathrm{H}_{2} \mathrm{O}_{2}$ for $15 \mathrm{~min}$ and incubated in avidin-biotin-peroxidase complex (1:200; Vector Laboratories) with $0.1 \%$ Triton X-100 overnight at $4^{\circ} \mathrm{C}$. Sections were then washed six times for $10 \mathrm{~min}$ in $0.1 \mathrm{M}$ PBS and then reacted with $0.025 \% 3,3^{\prime}$-diaminobenzidine (DAB) and $0.0008 \% \mathrm{H}_{2} \mathrm{O}_{2}$. Typically, nickel intensification (Adams, 1981) was performed with 2.5 mM nickel ammonium sulfate and $7 \mathrm{~mm}$ ammonium chloride during the $\mathrm{DAB} / \mathrm{H}_{2} \mathrm{O}_{2}$ incubation. Sections were then postfixed in osmium tetroxide $(0.1 \%$ in phosphate buffer) for $30 \mathrm{~min}$ and then dehydrated through an increasing series of ethanols. Last, sections were infiltrated overnight with a 50\%:50\% propylene oxide and epoxy resin mixture (Durcupan; Fluka), placed in a fresh $100 \%$ resin for several hours, flat embedded between glass slides and coverslips, and allowed to polymerize for $24 \mathrm{~h}$ in an oven at $60^{\circ} \mathrm{C}$.

Fluorescence immunocytochemistry for TH and DA. To assess the efficacy of 6-OHDA lesions, the midbrains of TH-Cre mice used for ex vivo studies were retained and kept in 4\% PFA and 15\% saturated picric acid $(\mathrm{v} / \mathrm{v})$ and later processed for TH immunoreactivity. Fifty to $60 \mathrm{~mm}$ coronal sections were cut in cold 0.15 M PBS using a Vibratome 1500 (Vibratome). Sections were cleared in 10\% methanol and 3\% hydrogen peroxide in $\mathrm{PBS}$ and washed three times in PBS. Blocking was done overnight at $4^{\circ} \mathrm{C}$ with $10 \%$ normal donkey serum (NDS) and $3 \%$ bovine serum albumin (BSA) with $0.5 \%$ Triton X-100 in PBS. Sections were then incubated in the same blocking solution at $4^{\circ} \mathrm{C}$ for $48 \mathrm{~h}$ containing rabbit anti-TH IgG (1:1500; catalog \#AB152; Millipore). After three $10 \mathrm{~min}$ washes in PBS, sections were incubated overnight at $4^{\circ} \mathrm{C}$ in donkey antirabbit IgG conjugated to Alexa Fluor 594 (1:500; catalog \#A-21207; Invitrogen) in PBS. After washing three times for $10 \mathrm{~min}$ in PBS, sections were mounted on slides and coverslipped with Vectashield hardset mounting medium (Vector Laboratories).

Visualization of DA and EGFP in the same neurons was performed in EGFP-TH mice and used a modified glutaraldehyde-based immunocytochemistry protocol (McRae-Degueurce and Geffard, 1986) after L-DOPA treatment. To inhibit peripheral metabolism of L-DOPA, the AADC inhibitor benserazide ( $15 \mathrm{mg} / \mathrm{kg}$, i.p.) was administered $30 \mathrm{~min}$ before L-DOPA injection (30 mg/kg, i.p.). Previous studies have shown that L-DOPA has a maximal effect on $[\mathrm{DA}]_{\mathrm{e}}$ levels $60 \mathrm{~min}$ after intraperitoneal administration (Zigmond et al., 1992; Miller and Abercrombie, 1999).

At $60 \mathrm{~min}$ after L-DOPA injection, heparin (4000 U/kg, i.p.) was administered, followed by ketamine/xylazine (160 and $30 \mathrm{mg} / \mathrm{kg}$, i.p., respectively). Anesthetized mice were then perfused transcardially with 20 $\mathrm{ml}$ of ice-cold buffer containing $0.1 \mathrm{M}$ sodium cacodylate and $1 \%(\mathrm{w} / \mathrm{v})$ sodium meta-bisulfate in double-distilled $\mathrm{H}_{2} \mathrm{O}\left(\mathrm{ddH}_{2} \mathrm{O}\right), \mathrm{pH}$ 6.2-6.5, followed by $100 \mathrm{ml}$ of fixative containing $5 \%$ glutaraldehyde, $1 \%$ sodium meta-bisulfate, and $0.1 \mathrm{~m}$ sodium cacodylate in $\mathrm{dd}_{2} \mathrm{O}, \mathrm{pH} 7.0-7.5$. The brain was then removed and left in the same fixative for 15-30 min and then washed four times with a $0.05 \mathrm{M}$ Tris-buffered solution containing $0.85 \%$ sodium meta-bisulfate (Tris-SMB) and sectioned at $50 \mu \mathrm{m}$ in the same solution with a vibratome. Sections were incubated in $0.1 \mathrm{M}$ sodium borohydride for $20 \mathrm{~min}$ in Tris-SMB and then washed four times for 10 min. Blocking was done at room temperature for $2 \mathrm{~h}$ with $5 \%$ NDS and $0.5 \%$ Triton X-100 solution in Tris-SMB. Sections were incubated for $16 \mathrm{~h}$ at $4^{\circ} \mathrm{C}$ in a polyclonal rabbit antibody against DA (catalog \#AB8888; Abcam) at a 1:2000 dilution in Tris-SMB containing 0.1\% Triton X-100 and $2 \%$ NDS. Sections were then washed three times for $10 \mathrm{~min}$ with Tris-SMB and incubated for $6 \mathrm{~h}$ at room temperature with donkey antirabbit secondary conjugated to Alexa Fluor 594 (1:400). Sections were washed and then mounted on slides in antifade solution, Vectashield hardest mounting medium (catalog \#H1400; Vector Laboratories), coverslipped, and sealed with nail polish.

Fluorescence immunocytochemistry for AADC. EGFP-TH mice were injected with heparin and anesthetized as described above. Mice were then perfused transcardially with $20 \mathrm{ml}$ of ice-cold artificial CSF, followed by $100 \mathrm{ml}$ of fixative containing $4 \% \mathrm{PFA}$ and $15 \%$ picric acid. The brains were then extracted and postfixed overnight in the same fixative. The following day, they were washed three times for $10 \mathrm{~min}$ in $0.15 \mathrm{M}$ PBS. The brains were then blocked, and $50-60 \mathrm{~mm}$ coronal sections were cut in cold $0.15 \mathrm{M}$ PBS using a vibratome. Sections were collected in six-well plates and cleared in 10\% methanol and 3\% hydrogen peroxide in PBS for 15-20 min. Blocking was done overnight at $4^{\circ} \mathrm{C}$ with $3 \%$ BSA and $10 \%$ NDS with $0.5 \%$ Triton X-100 in PBS. The sections were then incubated with $1 \%$ BSA and $1 \%$ NDS containing $0.1 \%$ Triton X-100 and a 1:2000 dilution of primary rabbit anti-DOPA decarboxylase (AADC) IgG (catalog \#ab3905; Abcam) in PBS for $1.5 \mathrm{~d}$ at room temperature. The sections were then washed three times for $10 \mathrm{~min}$ in PBS and incubated with 1:400 secondary donkey anti-rabbit IgG-Alexa Fluor 594 in PBS overnight at $4^{\circ} \mathrm{C}$ in the dark. Sections were then washed and mounted and coverslipped as above.

Fluorescence immunocytochemistry for VMAT2. Fluorescent immunocytochemistry for the VMAT2 was also performed. EGFP-TH mice were heparinized, anesthetized, perfused, postfixed, and sectioned as detailed above. Sections were collected in six-well plates and then treated with $1 \%$ sodium borohydride in PBS for $15 \mathrm{~min}$. The sections were then washed three times for $10 \mathrm{~min}$ in PBS. Sections were then incubated for $15 \mathrm{~min}$ in PBS containing 10\% methanol plus $3 \% \mathrm{H}_{2} \mathrm{O}_{2}$ (catalog \#216763; Sigma) and then washed in PBS three times for 10 min. Blocking was done overnight at $4^{\circ} \mathrm{C}$ with $3 \%$ BSA and $10 \%$ NDS with $0.5 \%$ Triton X-100 in PBS. The sections were then incubated in 1:2000 primary rabbit antiVMAT2 IgG (catalog \#20042; Immunostar) in PBS for $1.5 \mathrm{~d}$ at room temperature and then washed three times for $10 \mathrm{~min}$ in PBS. The sections were then incubated in 1:400 secondary donkey anti-rabbit IgG-Alexa Fluor 594 in PBS for $4-6 \mathrm{~h}$ at room temperature in the dark and washed for a final time, four times for $10 \mathrm{~min}$, in PBS. Sections were mounted on slides and coverslipped as above.

Fluorescence immunocytochemistry for the DAT. EGFP-TH mice were heparinized, anesthetized, perfused, postfixed, and sectioned as above. Sections were once again collected in six-well plates and then treated with $1 \%$ sodium borohydride in PBS for 15-20 min. The sections were then washed in PBS containing $10 \%$ methanol plus $3 \% \mathrm{H}_{2} \mathrm{O}_{2}$ (catalog \#216763; Sigma) for 15-20 min and then washed in PBS three times for 10 min. Blocking was done overnight at $4^{\circ} \mathrm{C}$ with $3 \%$ BSA and $10 \%$ NDS with $0.5 \%$ Triton X-100 in PBS. The sections were then incubated with 1:1000 primary rat anti-DAT IgG (catalog \#MAB369; Millipore Bioscience Research Reagents) in PBS for $1.5 \mathrm{~d}$ at room temperature and then washed three times in PBS for 10 min for each wash cycle. The sections were then incubated with 1:400 secondary donkey anti-rat IgG-Alexa Fluor 594 in PBS for $4-6 \mathrm{~h}$ at room temperature in the dark and washed for a final time, three times for $10 \mathrm{~min}$, in PBS, mounted, and coverslipped as above.

Statistical analysis. Numerical values for evoked $[\mathrm{DA}]_{\mathrm{e}}$ or IPSCs were reported as the mean \pm SEM. Significance of differences between group data were determined by either two-sample $t$ tests or one-way ANOVA, 


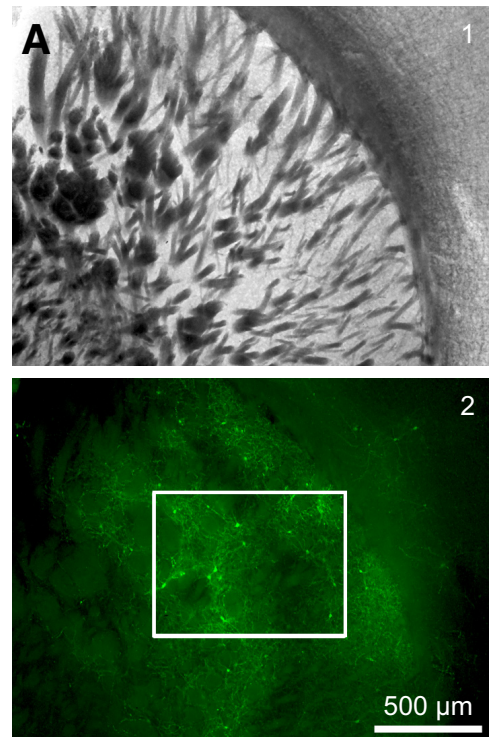

B

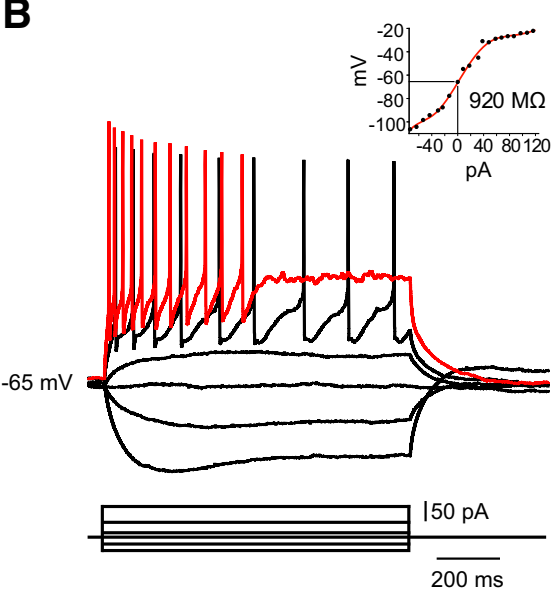

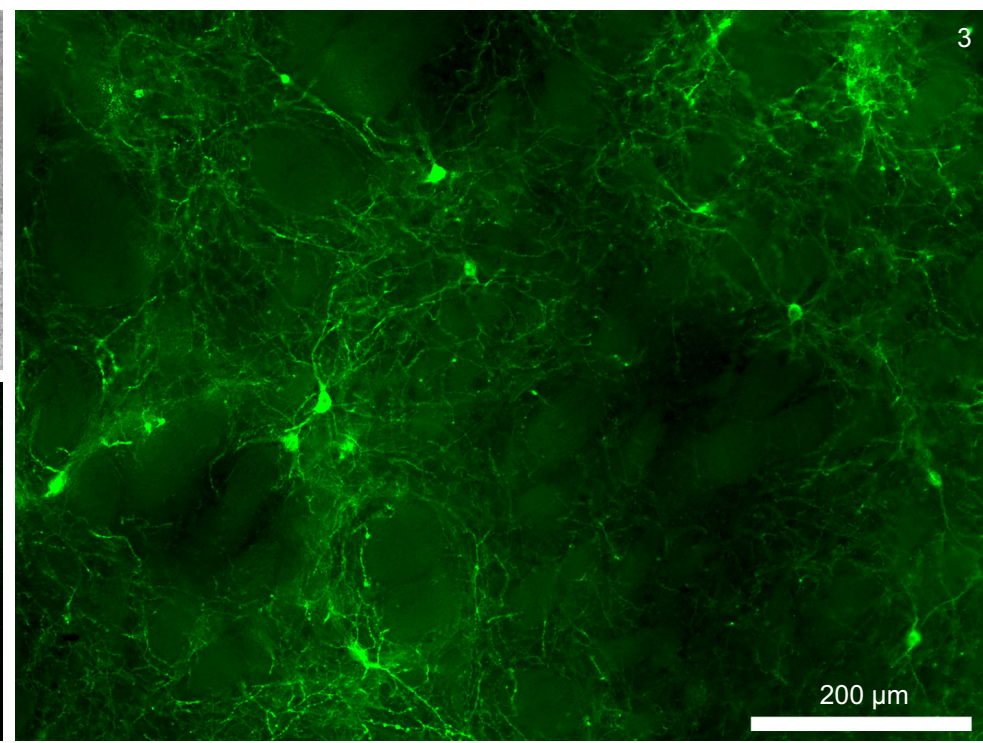

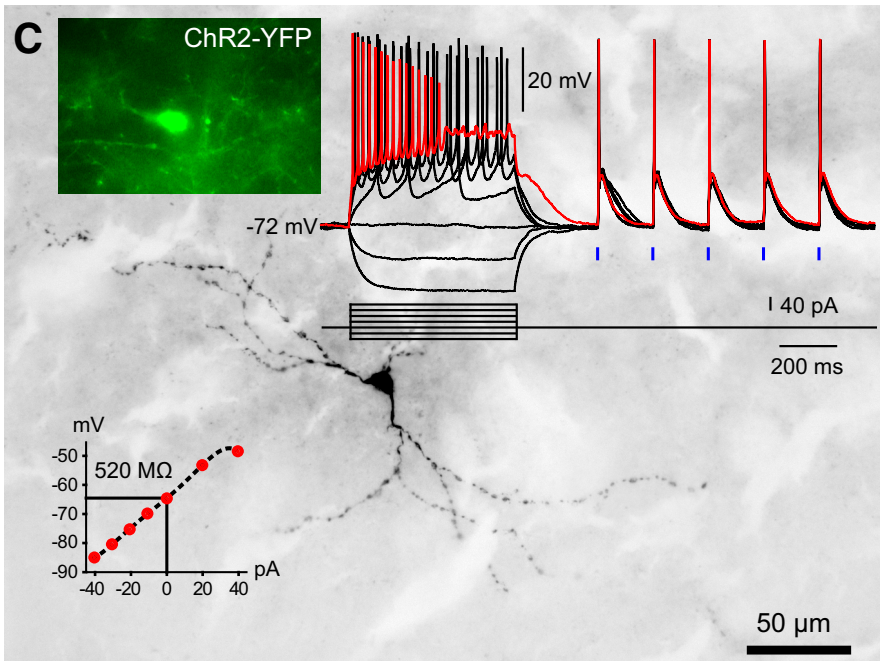

Figure 1. Optogenetic targeting and selective activation of striatal THINs. A1, Low-magnification bright-field photomicrograph of a coronal section through the striatum of a TH-Cre mouse, which was injected previously with AAV-ChR2-EYFP $(1.33 \mu \mathrm{l})$. A2, Fluorescence photomicrograph of the same field shown in $\boldsymbol{A} \mathbf{1}$ showing widespread viral transduction of striatal THINs. $\boldsymbol{A}$, Higher-magnification view of boxed area of $\boldsymbol{A 2}$, detailing abundant ChR2-EYFP somata, axons, and dendrites. $\boldsymbol{B}$, Representative whole-cell current-clamp recording of a type I striatal THIN from an EGFP-TH mouse, exhibiting typical high input resistance and the characteristic depolarization block at moderate depolarizing current injections typical of type I THINS reported previously. $C$, Whole-cell recording of a virally transduced ChR2-EYFP striatal THIN (top right inset) from a coronal striatal section from a TH-Cre mouse. Both the electrophysiology and anatomy were typical of type I striatal THINs as seen previously in EGFP-TH mice (compare with $\boldsymbol{B}$ ). Optical stimuli from a blue LED at $2.5 \mathrm{~ms}$ (blue ticks) evoke large depolarizations that consistently evoke spikes.

followed by Bonferroni's post hoc tests. Differences were considered to be significant if $p$ values were $<0.05$.

\section{Results}

Optogenetic targeting and activation of striatal THINs

We injected AAV-ChR2-EYFP bilaterally into the striata of 6-OHDA unilaterally lesioned TH-Cre mice. Two weeks after injection, there was widespread bilateral expression of the EYFP reporter in striatal somata and fibers, as shown in a typical striatum ipsilateral to 6-OHDA treatment in Figure $1 A$. Whole-cell current-clamp recordings and subsequent biocytin filling of ChR2-EYFP-labeled striatal THINs showed them to be indistinguishable in terms of both electrophysiology and morphology from striatal THINs identified previously in EGFP-TH mice (Ibáñez-Sandoval et al., 2010), as shown in Figure 1, B and C, for a typical type I striatal THIN.

Although quantitative stereology was not performed, compared with fluorescent striatal THINs in EGFP-TH mice, the Cre- mediated transduction seemed to revealed a higher density of these interneurons than reported previously (Ibáñez-Sandoval et al., 2010; Ünal et al., 2011). This may be related to the underreporting of $\mathrm{TH}$-immunoreactive neurons in the midbrains of EGFP-TH mice that we observed with DA immunofluorescence described below.

ChR2-EYFP axons arborized densely throughout the striatum and overlapped with the processes of neighboring THINs (Fig. 1A3). Whole-cell current-clamp recordings of these cells during presentation of single $2.5 \mathrm{~ms}$ blue light pulses demonstrated that striatal THINs were depolarized reliably and caused to fire action potentials by the optical stimulus, as shown in Figure $1 C$.

\section{Optogenetic targeting and activation of the nigrostriatal pathway and striatal DA release}

As a positive control for our optogenetic and voltammetric procedures, AAV-ChR2-EYFP was injected into the midbrain of TH-Cre mice, which transduced both mesencephalic dopami- 

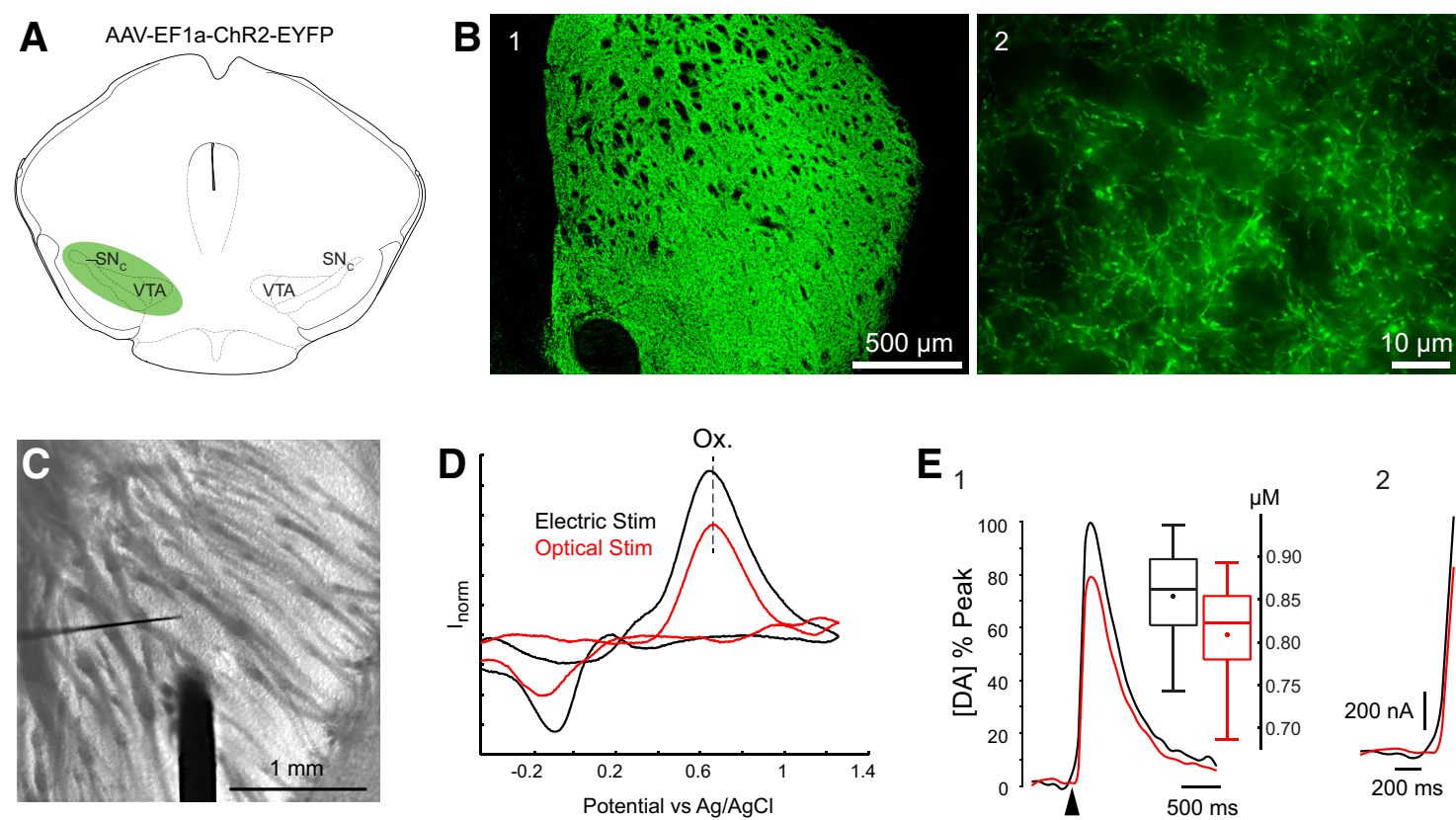

Figure 2. Voltammetric detection of optogenetically evoked striatal DA release from nigrostriatal neurons in a TH-Cre mouse. A, Schematic illustration of the midbrain injection site of AAV-ChR2-EYFP $(1.2 \mu$ l). B1, Coronal section of the striatum of a TH-Cre mouse 2 weeks after midbrain injection of AAV-ChR2-EYFP, showing an extremely dense distribution of fluorescent virally transduced nigrostriatal fibers throughout the striatum. B2, Higher-magnification photomicrograph of the same striatum shown in $\boldsymbol{B}$. C, Bright-field micrograph taken through the recording microscope shows the placement of the concentric bipolar stimulating electrode (thick dark structure) and the much thinner voltammetric CFE. D, Both electrical and optical stimulation protocols produce nearly identical cyclic voltammograms, whose peak oxidation ( $0 x$.) and reduction potentials identify them as resulting from DA. The dashed line shows the nearly identical oxidation potentials resulting from the two stimuli. E1, DA release after electrical (black) or optical (red) stimulation normalized to the maximum peak oxidation current. Box plot summaries of evoked [DA] from electrical (black) and optical (red) stimuli show no significant difference ( $n=11$ recordings). E2, A magnified view of the initial time profile of $\boldsymbol{E 1}$, illustrating identical release kinetics for both stimulation protocols. The arrowhead denotes the onset of electrical or optical stimulation.

nergic neurons and their striatal terminals as described previously (Tecuapetla et al., 2010). Two weeks later, coronal or parahorizontal sections of the striatum were cut, and epifluorescence imaging revealed widespread transduction of dopaminergic fibers throughout the striatum (Fig. 2B1). A stimulating electrode and carbon fiber probe were placed into striatal regions that were densely packed with ChR2-EYFP transduced nigrostriatal fibers (Fig. 2B2). After a single $250 \mu \mathrm{A}, 50 \mu$ s square electrical pulse or a single $2.5 \mathrm{~ms}$ blue light pulse, a large DA transient was evoked as shown in Figure 2D. DA transients evoked reliably by either optical or electrical stimuli exhibited very similar cyclic voltammograms. The mean peak DA concentration evoked by electrical stimulation (853.6 $\pm 36.1 \mathrm{~nm}$; range, $743-937 \mathrm{nM}$ ) was slightly, but not significantly, greater than that evoked by optical stimulation (808.5 \pm 40.7 nM; range, 687-893 nM; Fig. 2E1). There were also no significant differences between the mean voltages at which the oxidation and reduction peaks of DA evoked by the two stimuli occurred or any differences in the release kinetics of evoked DA, determined by the time to cover the $10-90 \%$ range, evoked by electrical (106 $\pm 3 \mathrm{~ms})$ or optical (105 $\pm 3 \mathrm{~ms})$ stimulation (two-sample $t$ test, $p>0.1 ; n=5$ slices, $n=2$ animals, $n=11$ recordings; Fig. 2E2).

Electrical and optogenetic activation of striatal THINs during simultaneous FSCV and whole-cell recording

Having shown that our voltammetric detection, viral transduction, and optogenetic techniques work in the midbrain, we set out to determine whether THINs could be stimulated to release DA. TH-Cre mice with unilateral 6-OHDA injections into the midbrain and bilateral striatal AAV-ChR2-EYFP injections were stimulated with a depolarizing current injection (50 pA for 500 $\mathrm{ms}$ ) in current-clamp mode, followed by a blue light pulse ( $5 \mathrm{~ms}$,
$750 \mathrm{~mW}$ LED; Fig. 3A1, blue triangle). To maximize the detection of any DA evoked from striatal THINs, the CFEs were always positioned within areas innervated densely with EYFP-labeled axons and within $50 \mu \mathrm{m}$ of the patched neuron. Although the current injection elicited a train of spikes and the single pulse of blue light elicited a triplet burst of spikes followed by a brief plateau potential that was terminated by a final spike, this activity did not elicit any detectable release of $[\mathrm{DA}]_{\mathrm{e}}$ (Fig. 3A2,A3). Next, EYFP $^{+}$THINs were then stimulated with a train of blue light pulses [ 105 -ms pulses with an interstimulus interval (ISI) of 25 ms; Fig. 3B1]. This optical train stimulation also failed to release any detectable DA (Fig. $3 B 2, B 3 ; n=5$ slices, $n=3$ animals, $n=$ 12 recordings).

\section{Optogenetic activation of striatal THINs and striatal DA release after lesioning}

To determine whether DA could be released from striatal THINs after unilateral 6-OHDA lesions, AAV-ChR2-EYFP was injected bilaterally into the striatum of $\mathrm{TH}-\mathrm{Cre}$ mice whose midbrains had been lesioned unilaterally by injection of 6-OHDA. This procedure transduces only striatal THINs, not the midbrain DA neurons or the nigrostriatal dopaminergic fibers. In addition, the unilateral lesions served two purposes. In numerous previous studies, the numbers of TH somata in the striatum increased after DA loss (Betarbet et al., 1997; Meredith et al., 1999; Huot and Parent, 2007), presumably as a result of an upregulation of TH activity in striatal TH neurons (Ünal et al., 2013). Thus, this afforded us the best chance to see DA evoked from striatal THINs. In addition, this also allowed us to have positive and negative controls for DA release in the striatum in the same slice.

Two weeks after injection, we performed ex vivo voltammetry in the striatum of both hemispheres, using local electrical or op- 

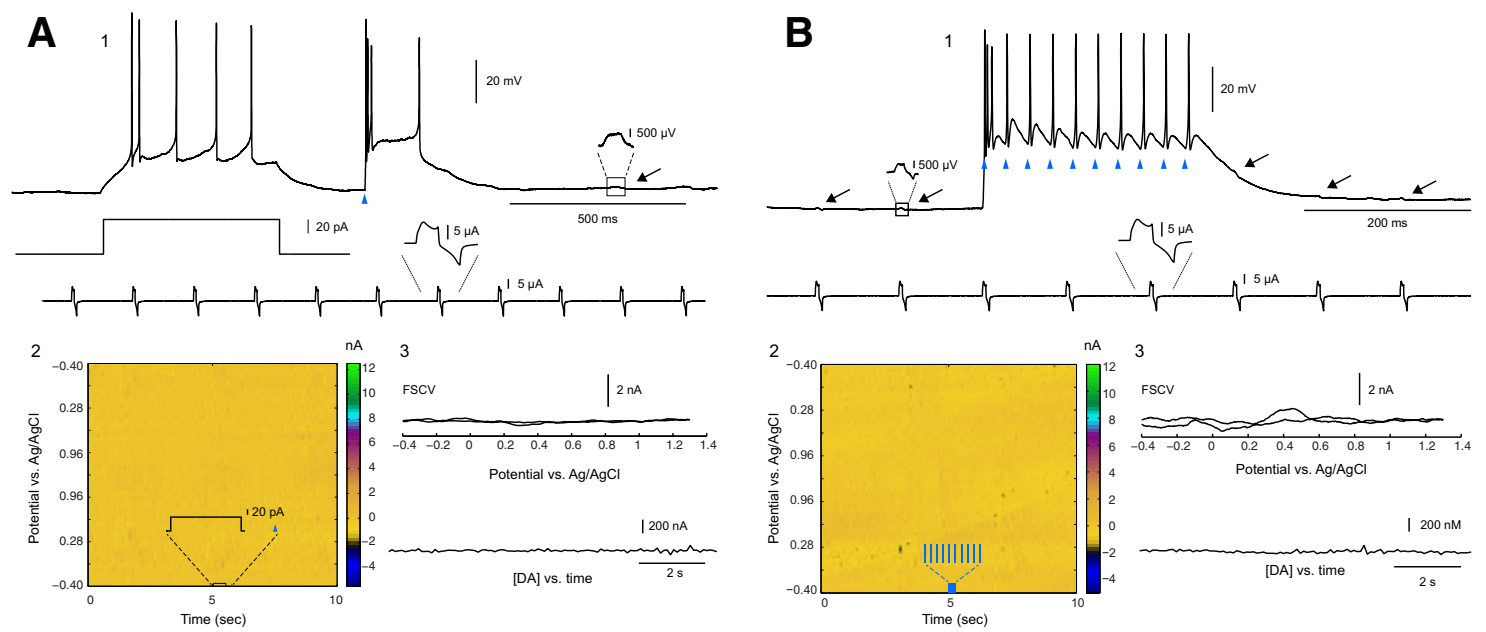

Figure 3. Optical activation of ChR2-YFP transduced striatal THINs in slices from mice with unilateral midbrain 6-OHDA lesions during simultaneous FSCV. A1, Neither current injection $(50 \mathrm{pA}$ for $500 \mathrm{~ms}$ ) nor optical stimulation (single $5 \mathrm{~ms}$ light pulse, blue triangle) elicited detectable evoked release of DA ipsilateral to midbrain 6-0HDA lesions. Note the absence of any oxidation or reduction peaks in the voltammetric heat map (A2). $\mathbf{A}$, No response in CFE (top) or any evoked DA release over a several-second-long recording period was detected $(n=$ 12 cells). $\boldsymbol{B}$, A strong optical stimulation train (10 5-ms blue light pulse with an ISI of $25 \mathrm{~ms}$ ) failed to evoke any detectable DA despite the elicitation of spikes to each light pulse (blue triangles). Black arrows in $\boldsymbol{B} 1$ indicate inductive voltage changes during current-clamp recording originating from the simultaneous voltammetric scan sweep below (see blow up of box region to the left of the recording sweep). $\mathbf{B} \boldsymbol{2}$ and $\mathbf{B} 3$ show the absence of any response in (FE or DA release.
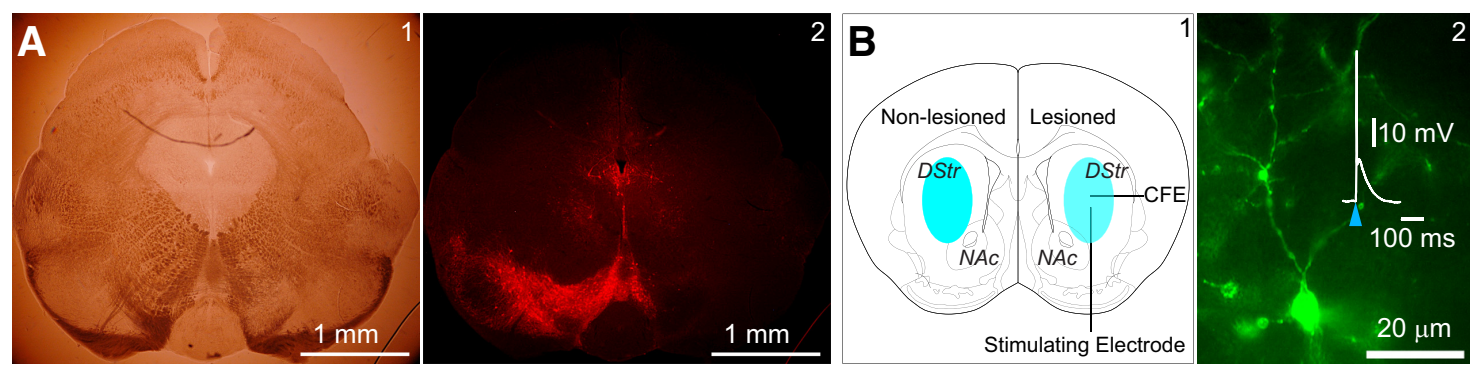

$\triangle$ Electrical stimulation $\triangle$ Optical stimulation
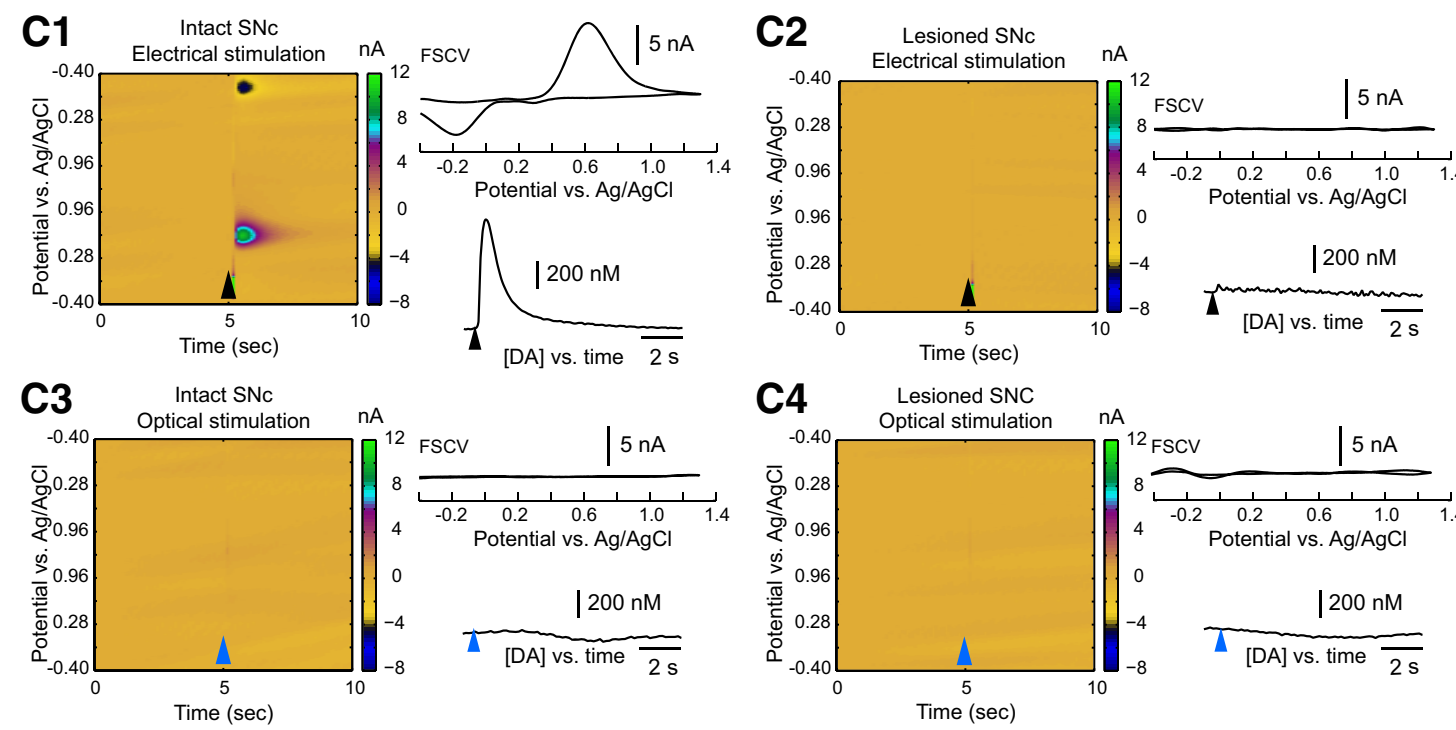

Figure 4. Simultaneous voltammetry in the striatum during optogenetic activation of striatal THINs. A1, Bright-field photomicrograph of a coronal section through the midbrain of a TH-Cre mouse after a unilateral midbrain 6-OHDA injection and bilateral striatal injections of AAV-ChR2-EYFP (1.33 $\mu$ l). A2, Fluorescent photomicrograph of the same field as in $\boldsymbol{A 1}$, processed for TH immun ofluorescence showing a complete absence of TH on the 6-OHDA-treated side but densely packed TH immunoreactive somata and processes in the substantia nigra and the ventral tegmental area on the contralateral side. B1, Schematic depiction of bilateral striatal injections of AAV-ChR2-EYFP. B2, Striatal THIN expressing ChR2-EYFP. The neuron reliably fires an action potential on a single 2.5 ms pulse of blue light. DStr, Dorsal striatum; NAc, nucleus accumbens. C, Voltammetric color maps, FSCVs, and [DA $]_{e}$ versus time plots. Electrical stimulation elicits DA from the control striatum (C1) but not from the lesioned side (C2). In contrast, optical stimulation failed to elicit any detectable amounts of DA from the striatum, either ipsilateral or contralateral to the 6-0HDA midbrain injection (C3, C4). Black and red arrowheads, respectively, denote the onset of electrical and optical stimulation ( $n=7$ mice, $n=13$ slices). 
A $10 \mu \mathrm{m}$ nomifensine $+10 \mu \mathrm{m}$ raclopride cocktail $\Delta$ Electrical stimulation $\Delta$ Optical stimulation
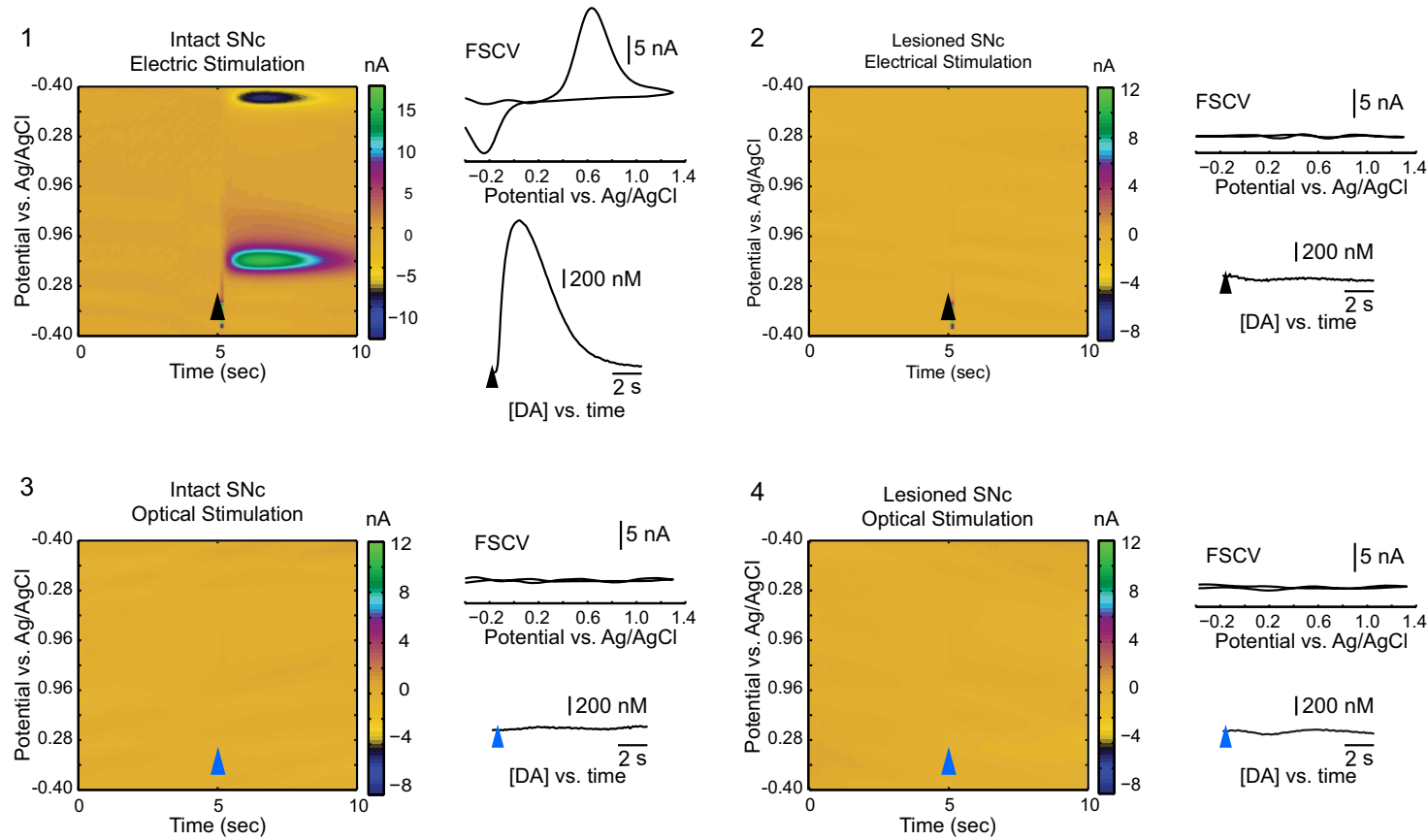

B

$\mathbf{C}_{\mu м}$
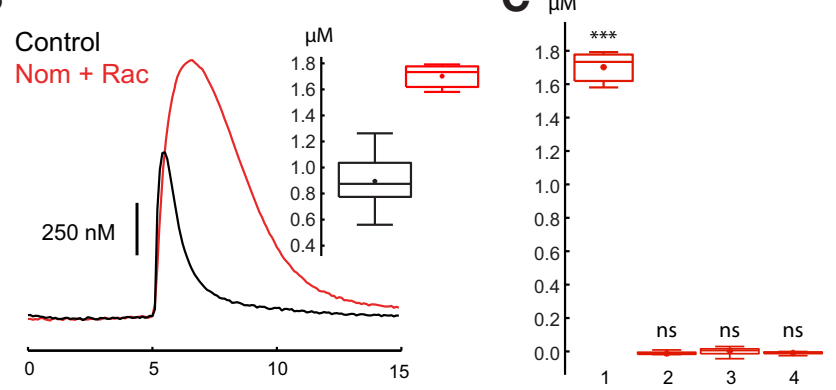

D

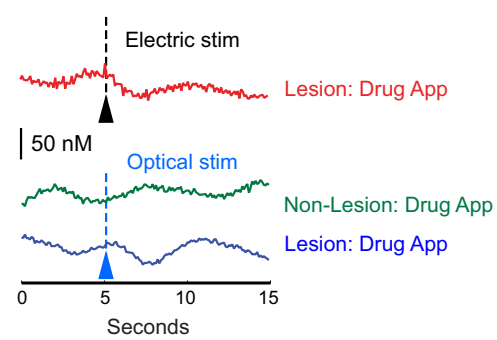

Figure 5. Effects of uptake and autoreceptor blockade on striatal DA release after optogenetic activation of striatal THINs. Nomifensine ( $10 \mu \mathrm{M})$ plus raclopride $(10 \mu \mathrm{M})$ present for all experiments ( $n=5$ mice, 14 slices). Slices from mice treated as described above. $\boldsymbol{A} \mathbf{1}$, Nomifensine plus raclopride greatly enhanced the release of DA in response to local electrical stimulation in the control striatum but failed to yield any detectable release of DA ipsilateral to the 6-OHDA midbrain injection (A2). $\boldsymbol{A 3}, \boldsymbol{A 4}, 0$ ptical stimulation failed to elicit detectable levels of DA from either the lesioned or control side. $B$, Overlay of evoked DA after electrical stimulation in the absence (black, $n=17$ recordings) and presence (red, $n=14$ recordings) of nomifensine (Nom) plus raclopride (Rac). Peak $[D A]$ in the presence of the drugs $(1.70 \pm 0.02 \mu \mathrm{m})$ was significantly greater than in their absence [0.96 $\left.\pm 0.05 \mu \mathrm{m} ; t_{(29)}=13.17, p<0.001\right]$. C, Box plot summaries of evoked peak [DA] in nomifensine plus raclopride for the following groups: electrical stimulation in striata contralateral to the lesion (group 1), electrical stimulation ipsilateral to the lesion (group 2), optical stimulation in the contralateral side (group 3), and optical stimulation ipsilateral to the lesion (group 4). D, Magnified scale of [DA] $]_{\mathrm{e}}$ versus time plots of $\boldsymbol{A 2}-\boldsymbol{A 4}$, showing no detectable DA for group 2 (red), group 3 (green), and group 4 (blue), even down at the level of CFE noise. ${ }^{* * *} p<0.001$.

Table 1. Full summary of all immunocytochemical results

\begin{tabular}{|c|c|c|c|c|c|c|c|c|c|c|}
\hline \multirow[b]{3}{*}{ Immunocytochemical marker } & \multirow{2}{*}{\multicolumn{2}{|c|}{$\underline{\text { Number of mice }}$}} & \multicolumn{4}{|c|}{$\left(\right.$ Marker + EGFP $\left.{ }^{+}\right) /$EGFP $^{+}$ratio } & \multicolumn{4}{|c|}{ Percentage colocalization } \\
\hline & & & \multicolumn{2}{|l|}{ Midbrain } & \multicolumn{2}{|c|}{ Striatum } & \multicolumn{2}{|c|}{ Midbrain } & \multicolumn{2}{|c|}{ Striatum } \\
\hline & Males & Females & SNC & VTA & Control & Lesion & SNC & VTA & Control & Lesion \\
\hline \multirow[t]{2}{*}{ DA } & 3 & 2 & $172 / 206$ & $85 / 128$ & $0 / 4630$ & $0 / 7905$ & $83.5 \%$ & $66.4 \%$ & $0 \%$ & $0 \%$ \\
\hline & & & $n=5$ & $n=5$ & $n=5$ & $n=5$ & $n=5$ & $n=5$ & $n=5$ & $n=5$ \\
\hline \multirow[t]{2}{*}{ AADC } & 2 & 3 & $164 / 200$ & $93 / 156$ & $0 / 4893$ & $0 / 7641$ & $82.0 \%$ & $59.6 \%$ & $0 \%$ & $0 \%$ \\
\hline & & & $n=5$ & $n=5$ & $n=5$ & $n=5$ & $n=5$ & $n=5$ & $n=5$ & $n=5$ \\
\hline \multirow[t]{2}{*}{ VMAT2 } & 2 & 3 & 157/195 & $88 / 143$ & $0 / 4254$ & $0 / 6733$ & $80.5 \%$ & $61.5 \%$ & $0 \%$ & $0 \%$ \\
\hline & & & $n=5$ & $n=5$ & $n=5$ & $n=5$ & $n=5$ & $n=5$ & $n=5$ & $n=5$ \\
\hline \multirow[t]{2}{*}{ DAT } & 2 & 2 & $127 / 158$ & $66 / 104$ & $0 / 5136$ & $0 / 7256$ & $80.4 \%$ & $63.4 \%$ & $0 \%$ & $0 \%$ \\
\hline & & & $n=4$ & $n=4$ & $n=4$ & $n=4$ & $n=4$ & $n=4$ & $n=4$ & $n=4$ \\
\hline
\end{tabular}

tical stimulation in each hemisphere $(n=7$ mice, $n=13$ bilateral slices). In all cases, the 6-OHDA injections were highly effective at lesioning the nigrostriatal projection on the ipsilateral side while leaving the dopaminergic input from the midbrain intact on the contralateral side. A typical example of a unilateral midbrain lesion is shown in Figure 4A2. The EYFP reporter in the transduced axons of the striatal THINs on the lesioned side served to guide the placement of the CFE directly in the region of the densest 

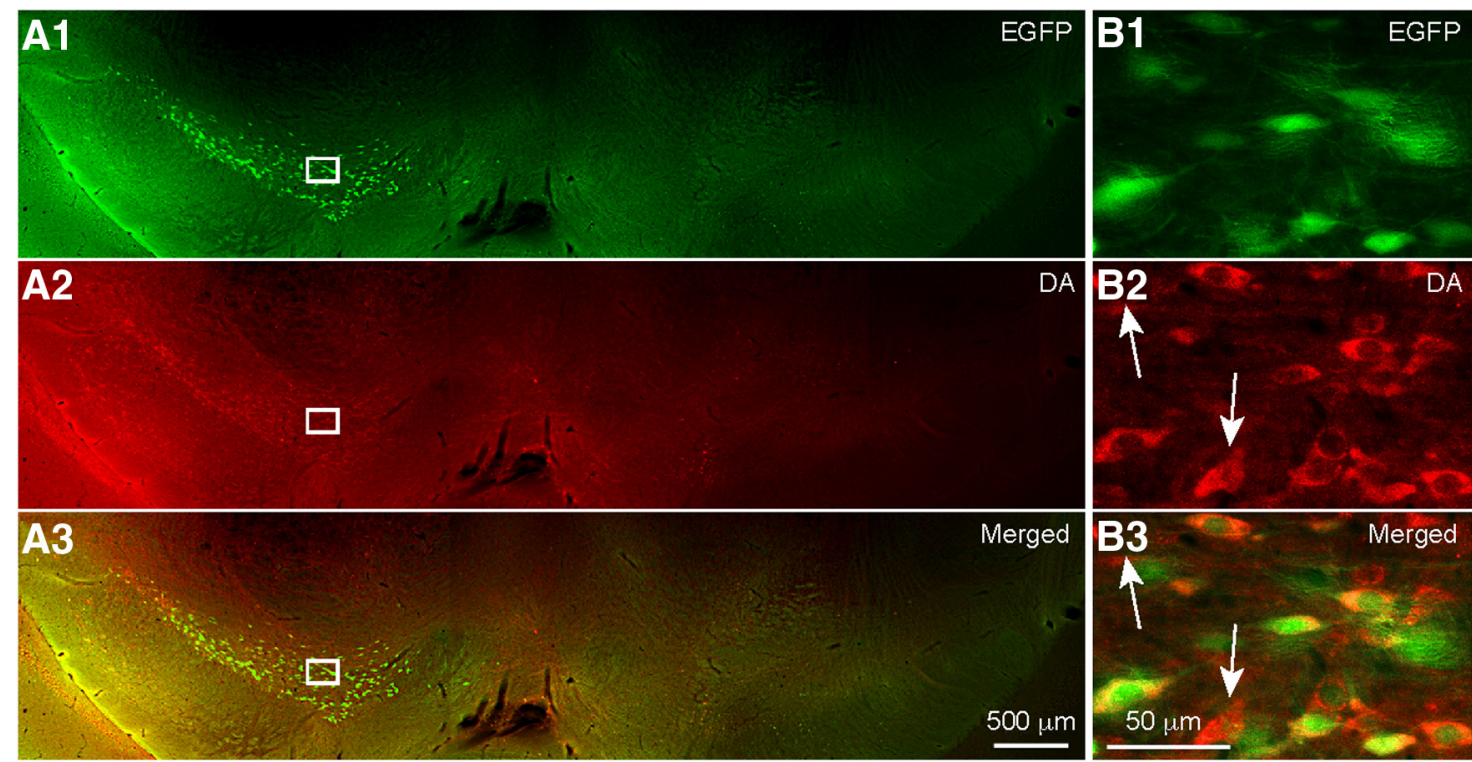

Figure 6. DA immunofluorescence in the midbrain of unilaterally lesioned EGFP-TH mice. A, Coronal section of the midbrain of an EGFP-TH mouse processed for DA immunofluorescence after injection of 6-OHDA into the right midbrain. Note the absence of both EGFP and DA immunofluorescence on the 6-OHDA-treated side but abundant EGFP and DA immunoreactive somata on the control side. $\boldsymbol{B}$, Higher-magnification photomicrographs of the corresponding boxed regions of $\boldsymbol{A}$ show DA immunofluorescent somata that colocalize EGFP $(n=5)$. In some cases, there were DA immunofluorescent somata that did not colocalize EGFP (white arrows), indicating that, in these mice, EGFP underreports the number of TH immunoreactive cells.

collection of striatal THINs and TH axons, as shown in Figure 4B2. Moreover, to verify that striatal THINs were firing in response to the optical stimuli, we performed whole-cell currentclamp recordings on striatal THINs. A typical example is shown in Figure 4B2, where a $2.5 \mathrm{~ms}$ optical stimulus evokes a large depolarization on which rides a single action potential.

Whereas local electrical stimulation $(250 \mu \mathrm{A}, 50 \mu \mathrm{s})$ elicited striatal DA release ( $850 \pm 60.2 \mathrm{~nm}$; range, $560.3 \mathrm{~nm}$ to $1.26 \mu \mathrm{M})$ contralateral to the 6-OHDA midbrain injections (Fig. 4C1), there was no detectable DA release in the ipsilateral striatum (Fig. $4 C 2$ ). Optical stimulation failed to elicit DA release from either striatum (Fig. 4C3,C4). This result indicates that the voltammetric detection of DA was working but that the DA release it detected on the contralateral side derived exclusively from the intact nigrostriatal fibers. Because the AAV-ChR2-EYFP injected into the striatum only transduced striatal TH neurons, these data demonstrate that striatal THINs do not release DA.

Next, we attempted to increase the levels of any potentially evoked $[D A]_{e}$ by bath application of $10 \mu \mathrm{M}$ nomifensine, a DA reuptake inhibitor, and $10 \mu \mathrm{M}$ raclopride, a $\mathrm{D}_{2}$ autoreceptor antagonist that markedly increases release of evoked DA release from midbrain dopaminergic neurons (Ogren et al., 1986; Earl et al., 1998; Robinson and Wightman, 2004) in similarly treated mice ( $n=5$ mice, $n=14$ slices). Compared with the control peak of $[D A]_{e}$ elicited by a single extracellular electrical stimulus in striatum contralateral to the midbrain lesion $(0.96 \pm 0.05 \mu \mathrm{M}$; range, $0.54-1.27 \mu \mathrm{M} ; n=17$ recordings), nomifensine plus raclopride significantly increased both the amplitude and duration of the DA transient, as illustrated in Figure 5A1 (1.70 $\pm 0.02 \mu \mathrm{M}$; range, $1.58-1.79 \mu \mathrm{M} ; n=14$ recordings; two-sample $t$ test, $p<$ $0.001)$. Despite this increase in electrically evoked $[D A]_{e}$ in the intact striatum, no electrically evoked DA was observed in the striatum ipsilateral to the midbrain lesion. Furthermore, despite DA uptake inhibition and autoreceptor blockade (Fig. 5C3,C4), optical stimulation failed to elicit any DA release in either striatum.

We repeated these experiments with $\mathrm{TH}-\mathrm{Cre}$ mice that were treated with L-DOPA (30 kg/mg, i.p.; $n=3)$ for $1 \mathrm{~h}$ before making our slice preparations and recording 40 min later. Electrically evoked DA release was unaltered contralateral to the lesion, and, as before, we failed to detect any evoked release of DA during optical stimulation in either side of the striatum. In these experiments, the typical noise level of our voltammetric recordings translated to a detectability level of $<25 \mathrm{~nm}[D A]_{e}$ (Fig. 5D).

Having shown that striatal THINs do not release DA, we attempted to determine why this is so. One possibility is that THINs do not contain DA or do but lack one or more of the other enzymes necessary for functional synthesis and/or release of DA. To determine this, striatal sections from EGFP-TH mice were examined for the expression of several traditional markers of dopaminergic neurons, including DA, AADC, VMAT2, and the DAT by fluorescence immunocytochemistry in mice injected unilaterally with 6-OHDA into the midbrain. As a positive control for all of our striatal immunofluorescence experiments, we used mice that had been injected unilaterally with 6-OHDA in the midbrain and examined both ipsilateral and contralateral midbrains and striata for all immunofluorescence experiments.

In general, for all the double-fluorescent labeling experiments, when the midbrain was examined the number of immunolabeled DA neurons was far greater than the number of EGFP-TH neurons. We first noticed this in our earliest experiments with TH immunolabeling of the midbrain several years ago (Ibáñez-Sandoval et al., 2010), but the mismatch has grown steadily larger over succeeding generations and will be apparent in many of the succeeding photomicrographs. The most probable explanation for this is epigenetic silencing of the transgene (Pikaart et al., 1998; Ellison and Kedes, 2014), which may accumulate transgenerationally as a result of incomplete germ-line erasure of imprinting (Kearns et al., 2000).

To try to partially control for these effects, we restricted our subjects to lineages to within two generations for each respective marker under investigation. As detailed further in the Discussion section, the presence of a subpopulation of monoenzymatic $\mathrm{AADC}^{+}$or $\mathrm{TH}^{+}$neurons in the midbrain that possess one or the other enzyme but not both have been reported previously (Ike- 


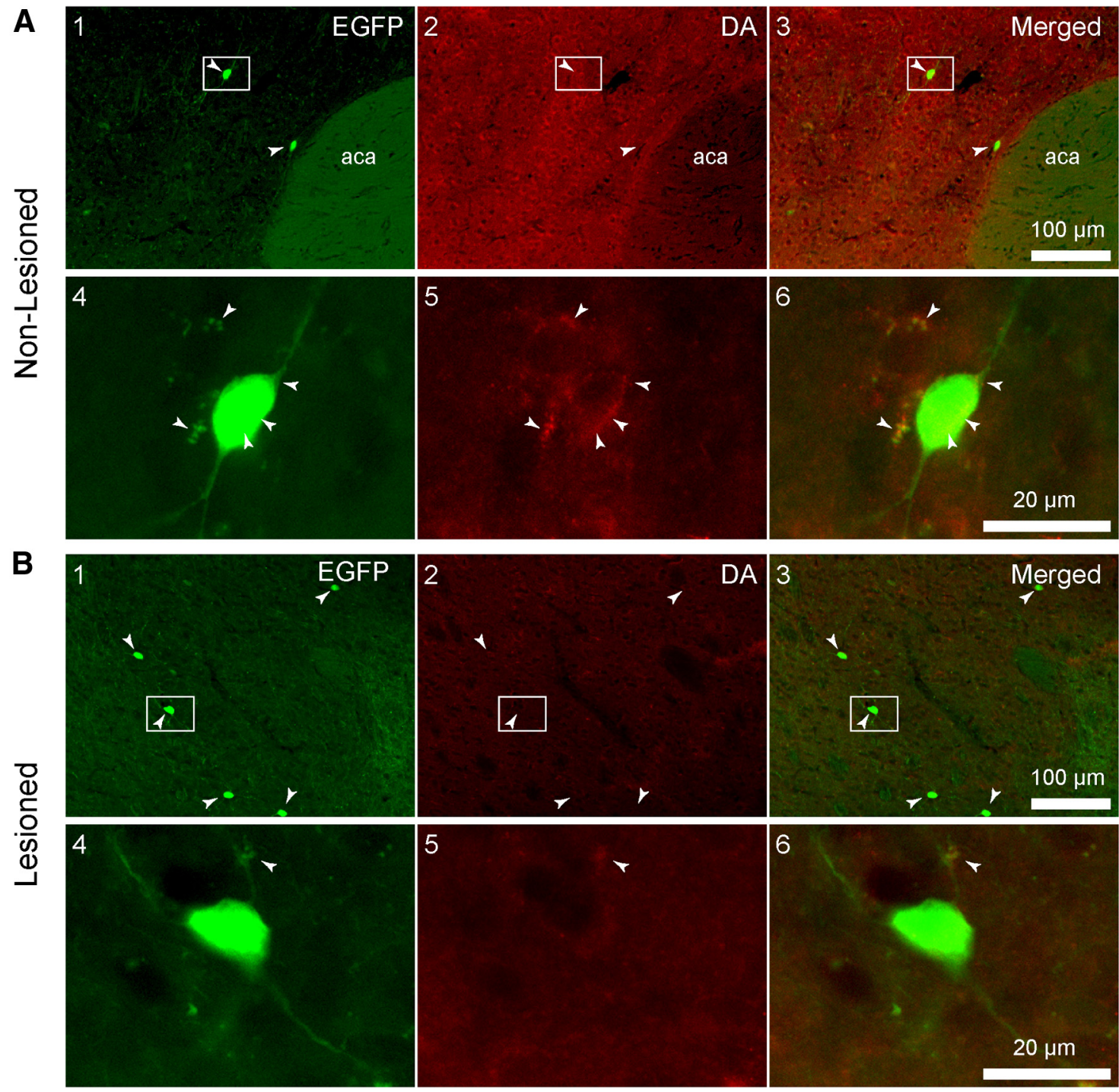

Figure 7. Photomicrographs of coronal sections through the dorsal striatum of an EGFP-TH mouse processed for DA immunofluorescence after unilateral 6-0HDA injections into the midbrain. $A$, Control side. $\boldsymbol{B}$, Lesioned side. Magnified views of the boxed regions in $\boldsymbol{A} \mathbf{A}-\mathbf{A} \mathbf{3}$ and $\boldsymbol{B} \mathbf{B}-\boldsymbol{B}$ are shown in $\boldsymbol{A 4}-\boldsymbol{A} \mathbf{6}$ and $\boldsymbol{B} \mathbf{B}-\boldsymbol{B} \mathbf{6}$. No colocalization of DA with EGFP-TH was evident in any of the sections examined ( $n=5$ brains). Note what appear to be DA immunoreactive puncta surrounding the THINs in $\mathbf{A} \boldsymbol{5}$ and $\boldsymbol{A} \boldsymbol{6}$ (white arrowheads), suggesting innervation of THINs by nigrostriatal terminals. Note the increase of visible THINs in the lesioned striatum (B1) compared with the nonlesioned striatum (B2; white arrowheads).

moto et al., 1998; Karasawa et al., 2007) could further exaggerate an apparent underreporting of EGFP-TH and simultaneously exaggerate the otherwise naturally occurring monoenzymatic expression of a given marker. However, it is important to keep in mind that we examined the colocalization of the respective markers with EGFP-TH with reasonably large sample size (Table 1) to ensure that, if any THINs expressed any of the markers, such colocalization would be detected.

\section{DA immunofluorescence in the midbrain and striata of EGFP-TH mice}

Examination of the midbrains $(n=5$ mice) revealed complete absence of neuronal somata expressing either EGFP or DA ipsilateral to the 6-OHDA injection, as shown in Figure 6A1. In contrast, on the contralateral side, there was robust expression of both DA immunofluorescence and EGFP fluorescence in densely packed somata and dendritic and axonal processes, shown in Figure 6B1-B3 at higher magnification. There was a considerable degree of overlap between EGFP fluorescence and DA immuno- fluorescence, but there were also a number of somata that expressed only one or the other of the two fluorophores (Fig. $6 B$, white arrows).

Overall, for the high-magnification regions inspected, there were 34 somata that were positive for both EGFP and DA of an average of $41 \mathrm{EGFP}^{+}$cells in total per $50 \mu \mathrm{m}$ section $(n=6$ sections, $n=5$ mice). Of the $206 \mathrm{EGFP}^{+}$cells inspected in the substantia nigra pars compacta ( $\mathrm{SNc}$ ), 172 colocalized DA, giving an $83.5 \%$ colocalization ratio (for full summary of all immunocytochemical results, see Table 1). This demonstrates that the DA immunofluorescence works and that, in dopaminergic neurons in EGFP-TH mice, there is colocalization of DA and EGFP.

In the striatum ipsilateral to the lesion, there appeared to be a small increase in the number of THINs compared with the contralateral side (Fig. $7 A, B$ ), as reported previously (Ünal et al., 2015). None of the striatal THINs on the ipsilateral $(n=7905$ cells) or the contralateral ( $n=4630$ cells) side expressed DA immunofluorescence ( $n=80$ sections). Interestingly, on the contralateral striata, we observed what appeared to be DA 


\section{A
$\frac{5}{\sqrt[0]{0}}$
$\frac{0}{}$
$\frac{0}{2}$}
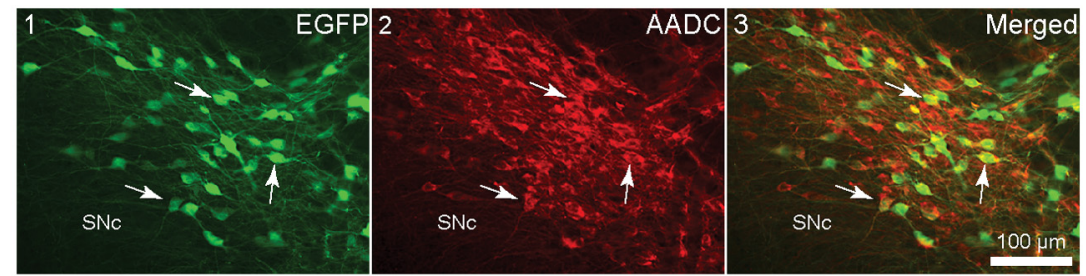

B
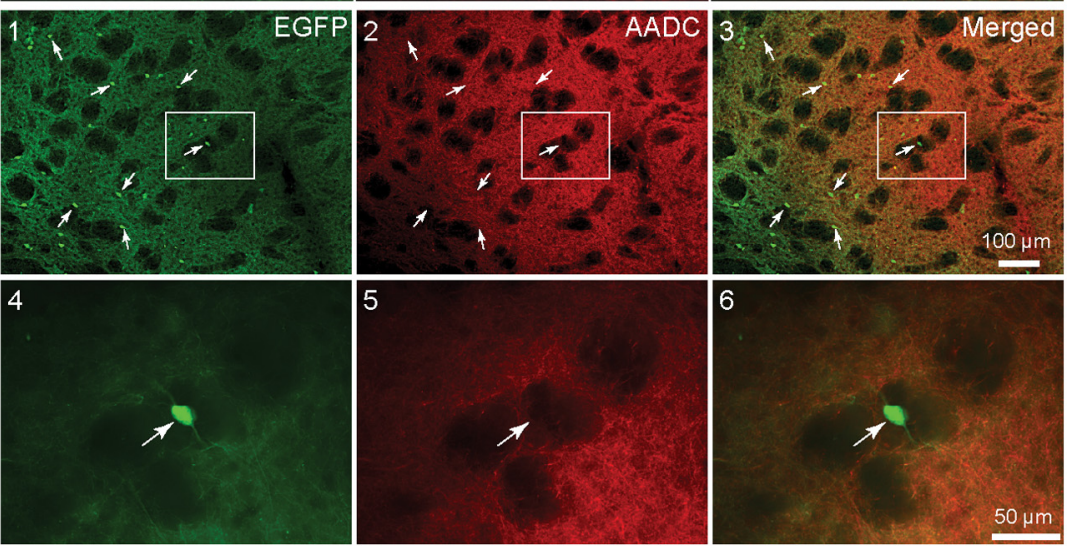

C
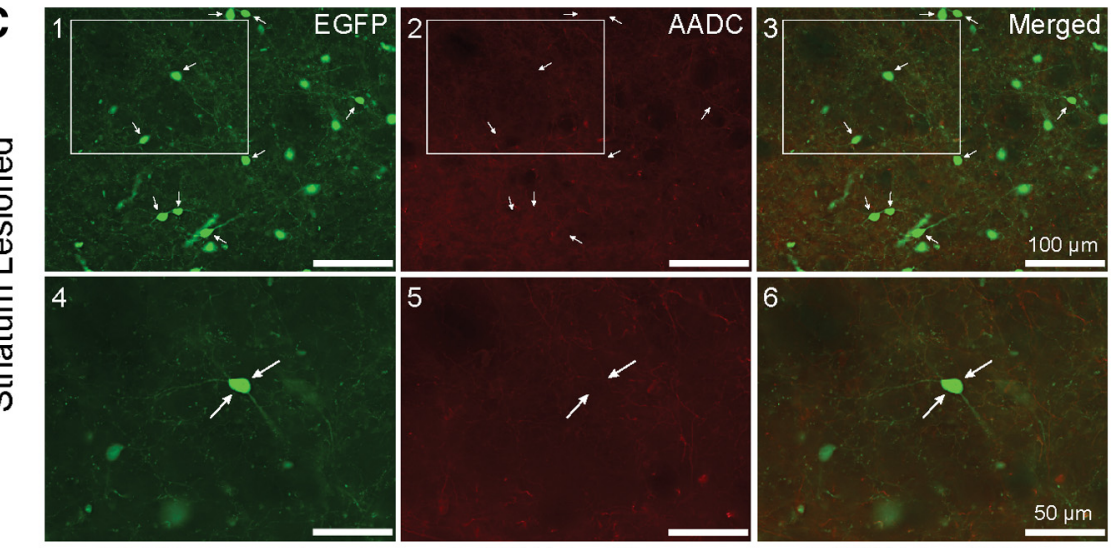

D
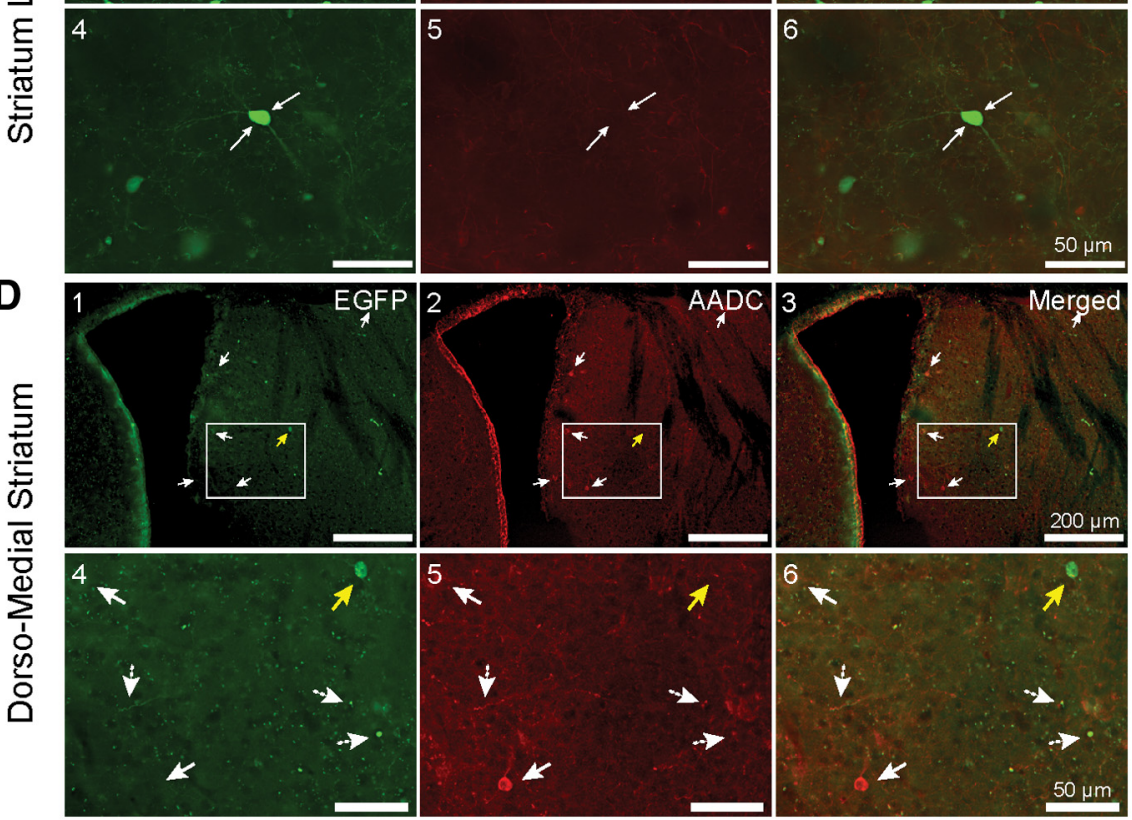

Figure 8. $A A D C$ immunofluorescence in the midbrain and striatum of unilaterally lesioned EGFP-TH mice. A, Highmagnification views of the contralateral midbrain showing EGFP-labeled neurons in the substantia nigra and VTA. White arrows point to representative neurons that colocalize EGFP and AADC. B1-B3, Low-magnification coronal photomicrographs of the striatum of an EGFP-TH mouse contralateral to the midbrain 6-OHDA injection that was processed for AADC immunofluorescence. Note that none of the THINs (white arrows) also express AADC. B4 -B6, High-magnification view of the boxed regions in $\boldsymbol{B 1}$ - $\boldsymbol{B} \mathbf{3}$ shows a single THIN that clearly lacks AADC expression. C, Photomicrographs of coronal sections of the striatum ipsilateral 6-OHDA injection into the midbrain. (4 -C6, Magnified view of the boxed regions in C1-C3 shows a representative THIN, which did not colocalize AADC. Note the increased expression of EGFP and lower AADC background expression on the lesioned side ( $\boldsymbol{C}$ compared with the control side $(\boldsymbol{B}) . \mathbf{D 1}-D \mathbf{D}, A A D C^{+}$-only striatal cells in the striatum. Fluorescent photomicrograph of a coronal section of the subcallosal region of the central striatum, near the lateral ventricle. Note a sparse number of $A A D C^{+}$-only cells (white arrows) distinct from EGFP ${ }^{+}$cells that do not colocalize AADC (yellow arrow). D4 -D6, Higher-magnification images of the boxed regions for D1-D3. immunofluorescent puncta surrounding $\mathrm{EGFP}^{+}$somata (Fig. 7A5,A6, white arrowheads). These were never observed ipsilateral to the midbrain 6-OHDA lesion (Fig. 7B5,B6) and are consistent with the innervation of THINs by nigrostriatal DA neurons (Ibáñez-Sandoval et al., 2015). We also noted a decreased level of background fluorescence in the lesioned striatum as a result of the loss of the nigrostriatal dopaminergic fibers. In three additional mice, L-DOPA was administered before they were killed as described in Materials and Methods. L-DOPA pretreatment did not affect the lack of expression of DA (data not shown).

\section{AADC immunofluorescence in the midbrain and striatum of EGFP-TH mice}

Figure 8 illustrates the positive control for the AADC antibody used in the midbrains of EGFP-TH mice $(n=5)$. AADC colocalized with EGFP throughout the midbrain. A representative coronal photomicrograph of the $\mathrm{SNc}$ is depicted in Figure $8 A$ that reveals numerous somata and their processes coexpressing AADC and EGFP ( $A 1-A 3$, white arrows). Of the $200 \mathrm{EGFP}^{+}$cells of the SNc, we found that 164 colocalized AADC, giving a colocalization ratio of $\approx 82 \%$ ( $n=6$ sections, $n=$ 5 mice).

The striatum was also examined for colocalization of EGFP and AADC. A small increase in the number of EGF$\mathrm{P}-\mathrm{TH}$ interneurons on the ipsilateral side was noted (Fig. 8, compare B1-B3 with C1-C3). Although there was strong staining for AADC in nigrostriatal fibers, no striatal EGFP interneurons were found to colocalize AADC of 4893 striatal EGFP ${ }^{+}$ interneurons in the contralateral side and 7641 interneurons in the ipsilateral side ( $n=71$ sections, $n=5$ mice).

\section{AADC-only striatal neurons}

The existence of a very small population of $\mathrm{AADC}^{+}$-only cells that differ in size and morphology from more numerous striatal $\mathrm{TH}^{+}$interneurons has been reported previously (Meredith et al., 1999). These cells were reported to exist in the subcallosal region of the rostral striatum. We also encountered a few of these cells and found that none of them colocalized EGFP. We found between 3 and 29 cells per $50 \mu \mathrm{m}$ coronal section. These cells averaged $11.7 \pm 1 \times 9 \pm 0.7 \mu \mathrm{m}(n=26$ cells, selected randomly from $n=4$ slices, $n=2$ mice), in agreement with the observations of Meredith et al. (1999). Figure $8 D$ depicts a low-magnification view of 

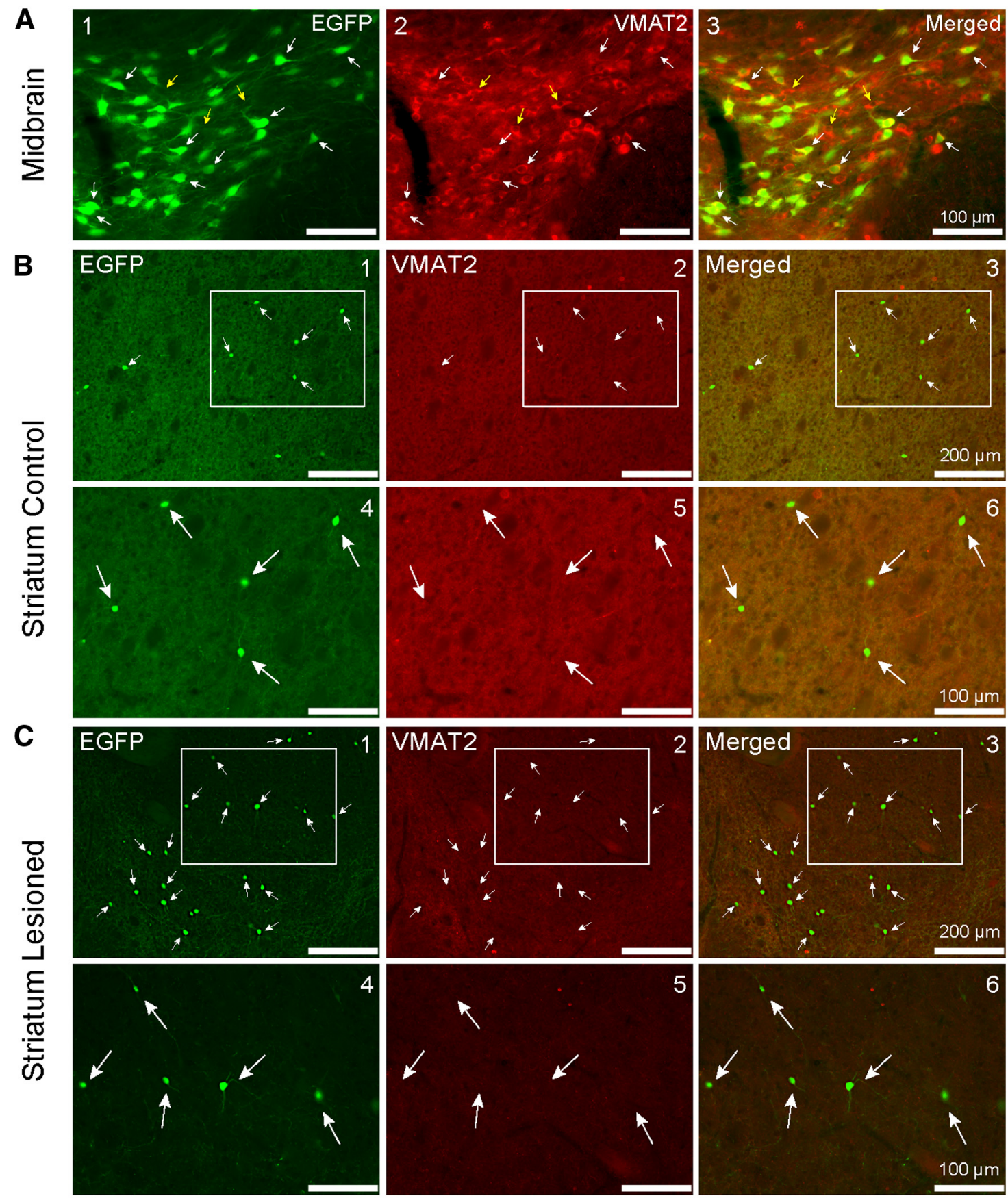

Figure 9. VMAT2 immunofluorescence in the midbrain of unilaterally lesioned EGFP-TH mice. A1-A3, Coronal photomicrographs of the SNc, depicting EGFP and VMAT2 immunopositive expression. Note the coexpression of EGFP and VMAT2 (white arrows) but also a few VMAT2-only cells that did not colocalize TH (yellow arrows). $\boldsymbol{B} 1-\boldsymbol{B} 3$, VMAT2 immunofluorescence in the striatum of unilaterally lesioned EGFP-TH mice. Low-magnification photomicrographs of a coronal section through the striatum of an EGFP-TH mouse contralateral to a midbrain 6-OHDA injection that was processed for VMAT2 immunofluorescence. Note that none of the EGFP-TH interneurons also express VMAT2. B4-B6, High-magnification view of the boxed regions in B1-B3 depicts a single THIN that clearly lacks VMAT2 expression. C1-C3, Photomicrographs of coronal sections of the striatum ipsilateral to 6-OHDA lesioning of the midbrain. C4 -C6, Magnified view of the boxed regions in $B 1-B 3$ shows a representative THIN that does not express VMAT2. Note the increased expression of EGFP and lower VMAT2 background expression on the lesioned side $(\boldsymbol{C})$ compared with the control side (B).

the subcallosal and periventricular regions of the striatum. There is one $\mathrm{AADC}^{+}$cell (representative example shown in D4-D6, white filled arrows) that does not also express EGFP (D4-D6, yellow arrows). Higher-magnification views of the boxed regions in Figure $8 D 1-D 3$ are shown in $D 4-D 6$, in which numerous EGFP nigrostriatal fibers and axonal blebs that also colocalize AADC (dashed white arrows) are visible. There was no significant difference between the number of AADC-only cells between the control and lesioned sides ( $n=6$ sections, $n=2$ mice). A total of $14.3 \pm 3.7$ cells were found in the control side and $11.8 \pm 2.7$ in the lesioned side (unpaired $t$ test, $p=0.59, \mathrm{df}=10$ ).

VMAT2 immunofluorescence in the midbrain and striatum of EGFP-TH mice

We next looked for expression of VMAT2 in striatal EGFP cells $(n=$ 5 mice). Numerous EGFP ${ }^{+}$large somata and their neurites colocal- 


\section{A}

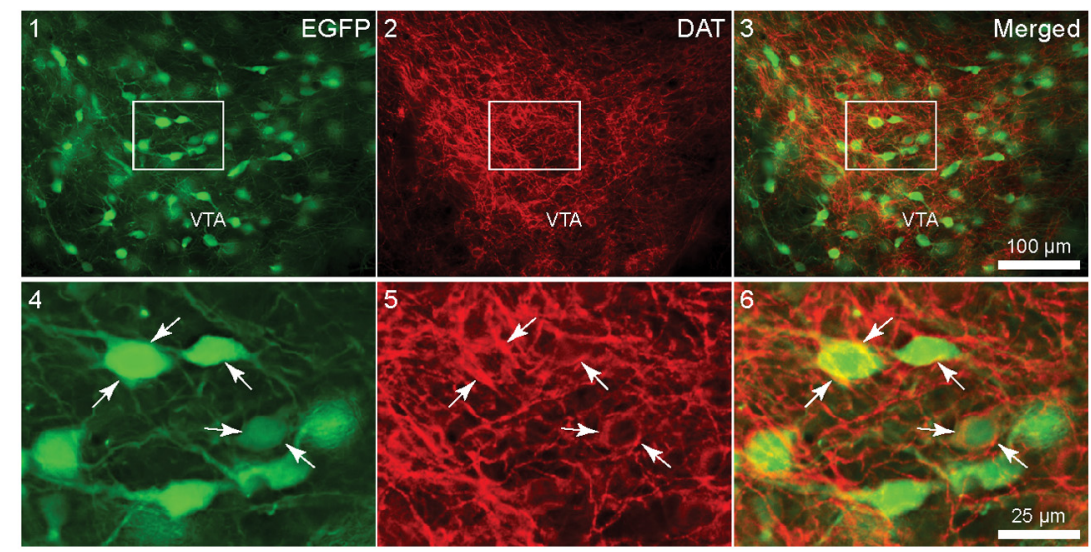

B
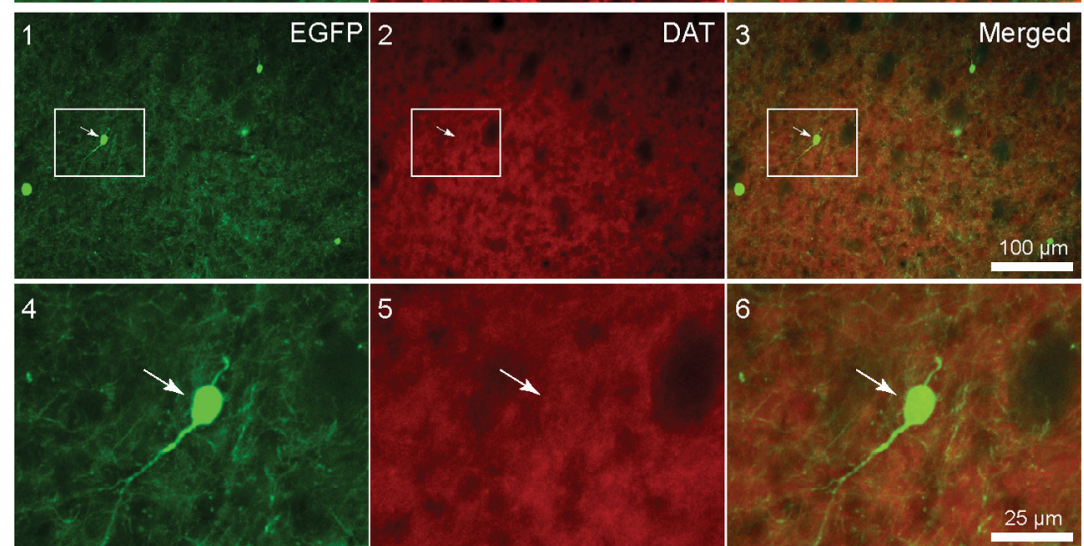

5

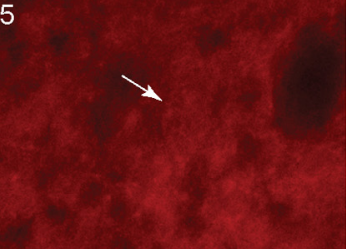

6

\section{C}
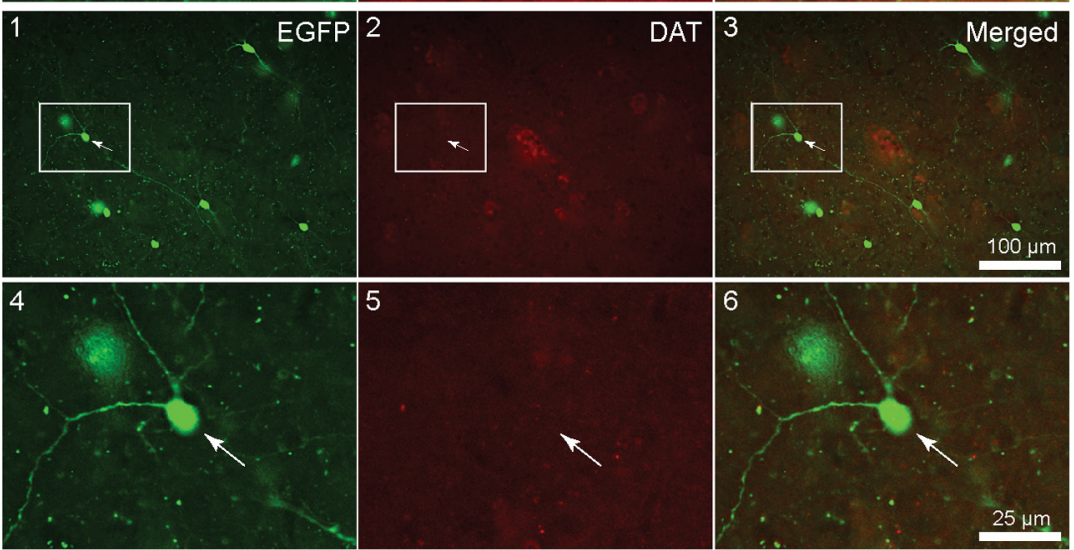

Figure 10. DAT immunofluorescence in the midbrain of unilaterally lesioned EGFP-TH mice. A1-A3, Low-magnification photomicrographs of a coronal section through the $\mathrm{SNc}$ contralateral to a midbrain injection of 6-OHDA showing a large number of EGFP fluorescent neurons. A4-A6, High-magnification photomicrograph of the boxed regions in A1-A3. Note the dense labeling of the neuropil and membrane of cells (white arrows). Note the perisomatic expression of AADC colocalizing with EGFP (A6) $B 1-B 3$, DAT immunofluorescence in the striatum of unilaterally lesioned EGFP-TH mice. Low-magnification photomicrograph of a coronal section through the striatum of an EGFP-TH mouse contralateral to a midbrain 6-OHDA injection that was processed for DAT immunofluorescence showing five EGFP-TH interneurons. $B 2, B 5$, Note the high background staining in the neuropil resulting from DAT expression in nigrostriatal axons. Merge indicates no coexpression of DAT and EGFP (B3). B4-B6, Highmagnification images of boxed regions in $\boldsymbol{B 1}$ - $\boldsymbol{B 3}$ show no expression of the DAT. C1-C3, Low-magnification photomicrograph of a coronal section through the striatum of an EGFP-TH mouse ipsilateral to a midbrain 6-OHDA injection that was processed for DAT immunofluorescence shows a number of EGFP-TH interneurons. Note that EGFP-TH does not colocalize with DAT immunofluorescence ( $\mathbf{C 4}-\mathbf{C 6}$ ). Note also the reduction in background staining compared with that in $\mathbf{C 5}$ compared with $\mathbf{B 5}$ as a result of the loss of the DAT-expressing nigrostriatal axons.

ized VMAT2 throughout the midbrain. A representative highmagnification photomicrograph is depicted in Figure $9 A$. In addition to the large number of EGFP cells that colocalized VMAT2 (white arrows), there was also a population of $\mathrm{VMAT2}^{+}$neurons that did not express EGFP (yellow arrows). Of the 88 highmagnification photographs imagined for the VTA, 88 of the 143
$\mathrm{EGFP}^{+}$cells colocalized VMAT2, yielding a colocalization ratio of $\approx 61.5 \%$ (Table 1 ).

In the striatum, we noted once again an increase of EGFP ${ }^{+}$cells on the lesioned side (Fig. 9C) compared with the nonlesioned side (Fig. 9B). There was also a marked reduction of VMAT2 staining in the neuropil in the lesioned version control side. None of the striatal THINs on the nonlesioned side (4254 cells) or any on the lesioned side (6733 cells) were found to colocalize VMAT2 $(n=65$ sections).

\section{DAT immunofluorescence in the midbrain and striatum of EGFP-TH mice}

We also tested for the DAT expression in EGFP-TH mice ( $n=4$; Fig. 10). In the midbrain, many of the EGFP ${ }^{+}$somata colocalized the DAT. Labeling was most intense at the periphery of the somata and proximal dendrites, as would be expected for a membrane-bound transporter (A4-A6, white arrows). Of the 158 EGFP $^{+}$somata, 127 colocalized the DAT in the SNc. This gave a colocalization ratio of $\approx 80.4 \%$.

In the striatum, none of the THINs examined on the control (5136 cells) or the lesioned (7256 cells) hemisphere were found to colocalize the DAT $(n=73 \mathrm{sec}-$ tions). Figure $10, B$ and $C$, shows representative coronal photomicrographs of the striatum of both the control and lesioned sides. There was both an increase in the apparent number of striatal EGFP ${ }^{+}$ cells and a decrease of DAT background staining in the neuropil of the lesioned side. For both the control and lesioned sides, high-magnification photomicrographs of representative THINs show that neither their somata nor their dendrites colocalize the DAT (Fig. 10B4-B6,C4-C6).

\section{Optogenetic activation of striatal \\ THINs leads to widespread inhibition of SPNs}

We showed previously that striatal THINs form powerful synaptic GABAergic connections onto SPNs (Ibáñez-Sandoval et al., 2010). This study was based on a relatively small number of paired recordings, in which THINS were found to evoke monosynaptic $\mathrm{GABA}_{\mathrm{A}}$ IPSPs/IPSCs in postsynaptic SPNs capable of delaying or blocking evoked spikes. To determine the effects of the synchronous activation of a large population of striatal THINs on SPNs, we performed whole-cell recordings of SPNs located within striatal regions innervated densely by the axons of virally transduced striatal THINs, which were abundant throughout the striatum (Fig. 11). In all cases ( $n=5$ mice, $n=11$ slices, $n=18$ SPNs), optogenetic activation of striatal THINs 
elicited GABA-mediated IPSPs sufficient to either delay the arrival of spiking or veto action potential firing altogether (Fig. 11A,B).

Pooling voltage-clamp data from paired recordings reported previously (Ibáñez-Sandoval et al., 2010) with new recordings showed that the mean amplitude of the unitary IPSC in SPNs (held at -45 to $-50 \mathrm{mV}$ ) after single action potentials evoked in THINs was $12.4 \mathrm{pA}$ $(n=5$ pairs; Fig. $11 C)$.

During synchronous activation of a larger population of virally transduced THINs after optical stimulation, the average IPSC on SPNs held at $-45 \mathrm{mV}$ was approximately three times as large (Fig. $11 D ; 31.7 \pm 4.9 \mathrm{pA}$; range, $25.5-58.0 \mathrm{pA}$; $n=12$ recordings).

\section{Discussion}

\section{Are striatal THINs dopaminergic?}

The first reports of striatal TH immunopositive neurons more than two decades ago generated considerable interest and quite a bit of controversy concerning their existence in normal animals of different species (Betarbet et al., 1997; Meredith et al., 1999; Lopez-Real et al., 2003; Mazloom and Smith, 2006; Darmopil et al., 2008), their identification as projection neurons or interneurons (Dubach et al., 1987; Tashiro et al., 1989), their number and morphology (Meredith et al., 1999; Mura et al., 2000; Palfi et al., 2002; Cossette et al., 2005a; Huot et al., 2007), and most importantly, whether or not they are actually dopaminergic. In particular, the consistent finding that the number of striatal TH immunoreactive neurons increases after loss of the nigrostriatal input in experimental PD models or in human PD (Dubach et al., 1987; Tashiro et al., 1989; Mura et al., 1995; Betarbet et al., 1997; Porritt et al., 2000; Cossette et al., 2005a; Huot et al., 2007) suggested that these neurons, often reported to be dopaminergic (Betarbet et al., 1997; Porritt et al., 2000; Palfi et al., 2002; Cossette et al., 2005a,b; Huot and Parent, 2007; San Sebastián et al., 2007), might play a compensatory role for decreased levels of striatal DA in idiopathic PD.

Most of these controversies were settled by the use of transgenic EGFP-TH mice that allowed us to identify striatal THINs in live slices and record and label them with biocytin, as well as to perform stereological estimates of their abundance (IbáñezSandoval et al., 2010; Ünal et al., 2011, 2015). These experiments showed that the THINs were medium sized, aspiny, or very sparsely spiny interneurons, expressed low levels of TH in their somata under control conditions, and made GABAergic synapses onto SPNs. However, the fact that they were functionally GABAergic did not eliminate the possibility that they might also be dopaminergic. The present results show that THINs are not immunoreactive for DA, even after treatments that maximize levels of TH activity and DA expression. Most importantly, ex vivo voltammetric studies using selective optogenetic stimulation of nigrostriatal fibers or THINs revealed that, although striatal DA release can be detected reliably after activation of nigrostriatal
B

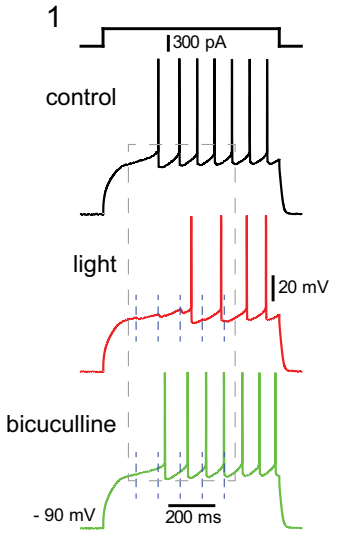

2

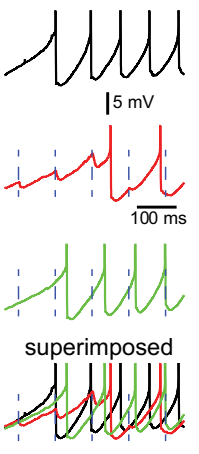

D

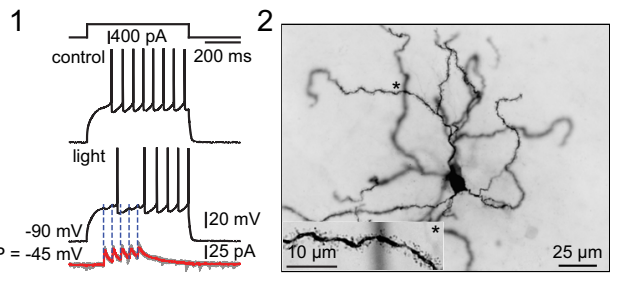

Figure 11. Striatal THINs exert widespread GABAergic inhibition onto SPNs. A, Ex vivo whole-cell current-clamp recording of an SPN in a TH-Cre mouse bilaterally injected with AAV-ChR2-EYFP in the striatum. Presentation of 2.5 ms blue light stimuli (blue (dic) elic IPSPS. B1, IPSPs in another SPN evoked by optical stimulation of THINs (dashed lines, red trace) are blocked by (black trace) were evoked in the same pair at a holding potential of $-45 \mathrm{mV}$. D1, Optogenetic activation of THINs during whole-cell recording of another SPN. Voltage-clamp recording of the same SPN during optogenetic activation of THINs reveals an average IPSC of $\sim 32 \mathrm{pA}$ at $-45 \mathrm{mV}$. D2, Photomicrograph of the SPN in D1. Inset at the bottom left is a magnified view of the dendrite marked by asterisks.

fibers of dopaminergic neurons, DA release was undetectable when striatal THINs were activated selectively. Thus, THINs do not release DA and are not dopaminergic neurons.

\section{Why are striatal THINs not dopaminergic?}

In the midbrain, for decades, TH expression has been accepted as proof that a neuron is dopaminergic. Why is the situation different in the striatum? The reason appears to be the lack of expression of several other enzymes required for DA synthesis and release.

First, these neurons do not express AADC and, therefore, not surprisingly, do not contain DA. Despite reports that at least some immunocytochemically identified striatal TH neurons also express the DAT (Betarbet et al., 1997; Cossette et al., 2005b; Tandé et al., 2006), none of the EGFP-TH interneurons in our study colocalized the DAT or VMAT2, responsible for cellular reuptake and vesicle packaging of DA, respectively. Although we cannot exclude the possibility that DAT is expressed in some striatal $\mathrm{TH}$ interneurons in primates but not rodents, it must be noted that the absence of DAT expression is consistent with the resistance of these interneurons (in all species) to destruction by either 6-OHDA or MPTP (Mazloom and Smith, 2006; Tandé et al., 2006; Darmopil et al., 2008). Both 6-OHDA and MPTP enter dopaminergic neurons via the DAT (Gainetdinov et al., 1997; Meredith and Rademacher, 2011; Bové and Perier, 2012; Hare et al., 2013). Thus, the resistance of striatal TH neurons to these neurotoxic compounds is almost certainly attributable to the lack of expression of the DAT and/or VMAT2.

Last, neurons termed "non-exocytotic catecholaminergic neurons" that express TH and/or AADC but that lack VMAT2 
have been identified in the striatum and elsewhere, as have neurons that express TH but not AADC or VMAT2 (Weihe et al., 2006), but these are clearly distinct from the EGFP-TH interneurons that were the focus of our experiments.

\section{Are any neurons in the striatum dopaminergic?}

Although we did not observe them in our material, small numbers of neurons immunoreactive for DA have been reported in both normal and lesioned striatum (Meredith et al., 1999; Mura et al., 2000). However, the size and morphology of these neurons differ from that of THINs observed either by EGFP fluorescence or after recording and biocytin labeling in our studies. Moreover, the abundance of these neurons is approximately one to two orders of magnitude less than that of the THINs in EGFP-TH mice, and their location was primarily restricted to the medial subcallosal region, whereas striatal THINs are distributed uniformly throughout the striatum (Ünal et al., 2011). Thus, there may exist a small population of DA-expressing striatal neurons, but they are clearly not the same neurons as the much more numerous THINs we have characterized here and elsewhere. Moreover, the fact that we observed, as others have reported (Meredith et al., 1999), a monoenzymatic $\mathrm{AADC}^{+}$population of cells that were markedly smaller in size and that did not colocalize $\mathrm{TH}$ further demonstrates that we were able to detect AADC in the striatum but that none of the much more numerous striatal EGF$\mathrm{P}-\mathrm{TH}^{+}$cells exhibited any coexpression with AADC. Thus, the smaller striatal $\mathrm{AADC}^{+}$monoenzymatic subpopulation and much larger $\mathrm{TH}^{+}$monoenzymatic population are distinct cell types.

Could this other very small population of "true" striatal dopaminergic neurons help compensate for the loss of striatal DA in PD? Because we performed voltammetric measurements throughout the striatum, including in the subcallosal area, and still detected no DA, then either there are no functional striatal dopaminergic neurons or the number of these neurons is so small that the amount of DA they release is below our $25 \mathrm{~nm}$ detection level. Naturally released DA during behavior has been measured in the tens to hundreds of nanomolar range in vivo (Robinson et al., 2002; Roitman et al., 2004; Beyene et al., 2010). Thus, any DA of striatal origin, even if extant, would be far below physiologically relevant levels.

In a recent study, Espadas et al. (2012) reported that they were able to measure DA of striatal origin evoked by local electrical stimulation with voltammetry after chronic L-DOPA treatment in 6-OHDA-lesioned animals. Although the possibility that striatal gene expression is altered by chronic L-DOPA treatment (Westin et al., 2001; El Atifi-Borel et al., 2009) cannot be excluded, given that a single nigrostriatal axon can occupy up to $5 \%$ of the striatal volume (Matsuda et al., 2009), the DA release observed more likely derived from incomplete 6-OHDA lesioning of nigrostriatal axons after intrastriatal injection of 6-OHDA (Espadas et al., 2012).

\section{What might be the function of TH in striatal THINs?}

There are many examples of CNS neurons that express some of the components of the DA phenotype but lack others (Weihe et al., 2006; for review, see Ugrumov, 2009). These include neurons that express $\mathrm{TH}$ but not AADC, neurons that express AADC but not $\mathrm{TH}$, and neurons that express both $\mathrm{TH}$ and AADC but lack VMAT2. It seems likely that most or all of the THINs that have been studied in EGFP-TH mice and those that are transduced in TH-Cre mice when AAV-ChR2-EYFP is injected into the striatum are monoenzymatic "DOPAergic" neurons, similar to $\mathrm{TH}$ immunopositive neurons in the hypothalamus that do not express DA even after administration of exogenous L-DOPA (Zoli et al., 1993). It has been suggested that, in the hypothalamus, DOPAergic neurons could supply L-DOPA non-exocytotically to nerve terminals of other neurons that do express AADC and thereby increase DA. Alternatively, perhaps L-DOPA itself plays some intracellular signaling role in the THINs (Ugrumov, 2009). Our results, plus those of others (Weihe et al., 2006; Ugrumov, 2009), raise the curious possibility that at least some of the many recently described populations of TH immunoreactive "dopaminergic" neurons in the midbrain that exhibit electrophysiological properties that are radically different (Roeper, 2013) than those of typical DA neurons (Grace and Onn, 1989) might be similar to the monoenzymatic striatal THINs.

\section{The striatal THIN network}

An additional finding that emerged from the optogenetic experiments was that all SPNs recorded responded to synchronous activation of THINs with inhibition. That indicates that the feedforward inhibitory network of THINs is widespread and, additionally, that both direct and indirect pathway SPNs must receive input from THINs. Whether there is any specificity or preferred target for the different THIN subtypes remains to be determined.

\section{Conclusions}

Although there probably are a very small number of dopaminergic neurons in the striatum, the abundant striatal THINs reported here and previous experiments (Ibáñez-Sandoval et al., 2010; Tepper et al., 2010; Ünal et al., 2011; Ünal et al., 2013; Luo et al., 2013) are not dopaminergic neurons. They are not immunoreactive for DA, AADC, VMAT2, or the DAT. Even under conditions that should optimize DA expression and synchronously activate a large proportion of the striatal THINs, DA release was undetectable. It is likely that the only part of the dopaminergic phenotype that THINs express is TH itself. The function of L-DOPA in THINs remains unknown, but one possibility is that it is supplied to monoenzymatic $\mathrm{AADC}^{+}$cells of the striatum or to the terminals of nigrostriatal dopaminergic neurons via the amino acid transporters (Ugrumov, 2009) and thereby contribute to DA release in a compromised striatum, although our voltammetric results suggest that this is unlikely to be the case.

All SPNs recorded were inhibited by optogenetic activation of striatal TH neurons after viral transduction with ChR2. Therefore, THINs represent a unique class of striatal GABAergic interneurons that produce widespread and effective inhibition on both direct and indirect pathway SPNs throughout the striatum.

\section{References}

Adams JC (1981) Heavy metal intensification of DAB-based HRP reaction product. J Histochem Cytochem 29:775. CrossRef Medline

Betarbet R, Turner R, Chockkan V, DeLong MR, Allers KA, Walters J, Levey AI, Greenamyre JT (1997) Dopaminergic neurons intrinsic to the primate striatum. J Neurosci 17:6761-6768. Medline

Beyene M, Carelli RM, Wightman RM (2010) Cue-evoked dopamine release in the nucleus accumbens shell tracks reinforcer magnitude during intracranial self-stimulation. Neuroscience 169:1682-1688. CrossRef Medline

Bové J, Perier C (2012) Neurotoxin-based models of Parkinson's disease. Neuroscience 211:51-76. CrossRef Medline

Cossette M, Parent A, Lévesque D (2004) Tyrosine hydroxylase-positive neurons intrinsic to the human striatum express the transcription factor Nurr1. Eur J Neurosci 20:2089-2095. CrossRef Medline

Cossette M, Lecomte F, Parent A (2005a) Morphology and distribution of 
dopaminergic neurons intrinsic to the human striatum. J Chem Neuroanat 29:1-11. CrossRef Medline

Cossette M, Lévesque D, Parent A (2005b) Neurochemical characterization of dopaminergic neurons in human striatum. Parkinsonism Relat Disord 11:277-286. CrossRef Medline

Darmopil S, Muñetón-Gomez VC, de Ceballos ML, Bernson M, Moratalla R (2008) Tyrosine hydroxylase cells appearing in the mouse striatum after dopamine denervation are likely to be projection neurones regulated by L-DOPA. Eur J Neurosci 27:580-592. CrossRef Medline

Dubach M, Schmidt R, Kunkel D, Bowden DM, Martin R, German DC (1987) Primate neostriatal neurons containing tyrosine hydroxylase: immunohistochemical evidence. Neurosci Lett 75:205-210. CrossRef Medline

Earl CD, Sautter J, Xie J, Kruk ZL, Kupsch A, Oertel WH (1998) Pharmacological characterisation of dopamine overflow in the striatum of the normal and MPTP-treated common marmoset, studied in vivo using fast cyclic voltammetry, nomifensine and sulpiride. J Neurosci Methods 85: 201-209. CrossRef Medline

El Atifi-Borel M, Buggia-Prevot V, Platet N, Benabid AL, Berger F, SgambatoFaure V (2009) De novo and long-term l-Dopa induce both common and distinct striatal gene profiles in the hemiparkinsonian rat. Neurobiol Dis 34:340-350. CrossRef Medline

Ellison TJ, Kedes DH (2014) Variable episomal silencing of a recombinant herpesvirus renders its encoded GFP an unreliable marker of infection in primary cells. PLoS One 9:e111502. CrossRef Medline

English DF, Ibanez-Sandoval O, Stark E, Tecuapetla F, Buzsáki G, Deisseroth K, Tepper JM, Koos T (2012) GABAergic circuits mediate the reinforcement-related signals of striatal cholinergic interneurons. Nat Neurosci 15:123-130. Medline

Espadas I, Darmopil S, Vergaño-Vera E, Ortiz O, Oliva I, Vicario-Abejón C, Martín ED, Moratalla R (2012) L-DOPA-induced increase in THimmunoreactive striatal neurons in parkinsonian mice: insights into regulation and function. Neurobiol Dis 48:271-281. CrossRef Medline

Gainetdinov RR, Fumagalli F, Jones SR, Caron MG (1997) Dopamine transporter is required for in vivo MPTP neurotoxicity: evidence from mice lacking the transporter. J Neurochem 69:1322-1325. Medline

Gerfen CR, Wilson CJ (1996) The basal ganglia. In: Handbook of chemical neuroanatomy, Ed 12 (Swanson LW, Bjorklund A, Hokfelt T, eds), pp 371-468. Amsterdam: Elsevier Science.

Gittis AH, Kreitzer AC (2012) Striatal microcircuitry and movement disorders. Trends Neurosci 35:557-564. CrossRef Medline

Gittis AH, Nelson AB, Thwin MT, Palop JJ, Kreitzer AC (2010) Distinct roles of GABAergic interneurons in the regulation of striatal output pathways. J Neurosci 30:2223-2234. CrossRef Medline

Gong S, Zheng C, Doughty ML, Losos K, Didkovsky N, Schambra UB, Nowak NJ, Joyner A, Leblanc G, Hatten ME, Heintz N (2003) A gene expression atlas of the central nervous system based on bacterial artificial chromosomes. Nature 425:917-925. CrossRef Medline

Grace AA, Onn SP (1989) Morphology and electrophysiological properties of immunocytochemically identified rat dopamine neurons recorded in vitro. J Neurosci 9:3463-3481. Medline

Hare DJ, Adlard PA, Doble PA, Finkelstein DI (2013) Metallobiology of 1-methyl-4-phenyl-1,2,3,6-tetrahydropyridine neurotoxicity. Metallomics 5:91-109. CrossRef Medline

Huot P, Parent A (2007) Dopaminergic neurons intrinsic to the striatum. J Neurochem 101:1441-1447. CrossRef Medline

Ibáñez-Sandoval O, Tecuapetla F, Unal B, Shah F, Koós T, Tepper JM (2010) Electrophysiological and morphological characteristics and synaptic connectivity of tyrosine hydroxylase-expressing neurons in adult mouse striatum. J Neurosci 30:6999-7016. CrossRef Medline

Ibáñez-Sandoval O, Tecuapetla F, Unal B, Shah F, Koós T, Tepper JM (2011) A novel functionally distinct subtype of striatal neuropeptide y interneuron. J Neurosci 31:16757-16769. CrossRef Medline

Ibáñez-Sandoval O, Shah F, Tepper JM, Koós T (2015) Dopaminergic and cholinergic modulation of striatal tyrosine hydroxylase interneurons. Neuropharmacology, in press.

Ikemoto K, Nagatsu I, Nishimura A, Nishi K, Arai R (1998) Do all of human midbrain tyrosine hydroxylase neurons synthesize dopamine? Brain Res 805:255-258. CrossRef Medline

John CE, Jones SR (2007) Fast scan cyclic voltammetry of dopamine and serotonin in mouse brain slices. In: Electrochemical methods for neuroscience, Chap 4 (Michael AC, Borland LM, eds). Boca Raton, FL: CRC.
Karasawa N, Hayashi M, Yamada K, Nagatsu I, Iwasa M, Takeuchi T, Uematsu M, Watanabe K, Onozuka M (2007) Tyrosine hydroxylase (TH)and aromatic-L-amino acid decarboxylase (AADC)-immunoreactive neurons of the common marmoset (Callithrix jacchus) brain: an immunohistochemical analysis. Acta Histochem Cytochem 40:83-92. CrossRef Medline

Kawaguchi Y (1993) Physiological, morphological, and histochemical characterization of three classes of interneurons in rat neostriatum. J Neurosci 13:4908-4923. Medline

Kearns M, Preis J, McDonald M, Morris C, Whitelaw E (2000) Complex patterns of inheritance of an imprinted murine transgene suggest incomplete germline erasure. Nucleic Acids Res 28:3301-3309. CrossRef Medline

Klaus A, Planert H, Hjorth JJ, Berke JD, Silberberg G, Kotaleski JH (2011) Striatal fast-spiking interneurons: from firing patterns to postsynaptic impact. Front Syst Neurosci 5:57. Medline

Koós T, Tepper JM (1999) Inhibitory control of neostriatal projection neurons by GABAergic interneurons. Nat Neurosci 2:467-472. CrossRef Medline

Koós T, Tepper JM (2002) Dual cholinergic control of fast-spiking interneurons in the neostriatum. J Neurosci 22:529-535. Medline

Lopez-Real A, Rodriguez-Pallares J, Guerra MJ, Labandeira-Garcia JL (2003) Localization and functional significance of striatal neurons immunoreactive to aromatic L-amino acid decarboxylase or tyrosine hydroxylase in rat Parkinsonian models. Brain Res 969:135-146. CrossRef Medline

Luo R, Janssen MJ Partridge JG, Vicini S (2013) Direct and GABA-mediated indirect effects of nicotinic $\mathrm{ACh}$ receptors on striatal neurones. J Physiol 59:203-217. CrossRef

Mao L, Lau YS, Petroske E, Wang JQ (2001) Profound astrogenesis in the striatum of adult mice following nigrostriatal dopaminergic lesion by repeated MPTP administration. Brain Res Dev Brain Res 131:57-65. CrossRef Medline

Matsuda W, Furuta T, Nakamura KC, Hioki H, Fujiyama F, Arai R, Kaneko T (2009) Single nigrostriatal dopaminergic neurons form widely spread and highly dense axonal arborizations in the neostriatum. J Neurosci 29:444-453. CrossRef Medline

Mazloom M, Smith Y (2006) Synaptic microcircuitry of tyrosine hydroxylase-containing neurons and terminals in the striatum of 1-methyl-4-phenyl-1,2,3,6-tetrahydropyridine-treated monkeys. J Comp Neurol 495:453-469. CrossRef Medline

McRae-Degueurce A, Geffard M (1986) One perfusion mixture for immunocytochemical detection of noradrenaline, dopamine, serotonin and acetylcholine in the same rat brain. Brain Res 376:217-219. CrossRef Medline

Mehler WR (1981) The basal ganglia-circa 1982. A review and commentary. Appl Neurophysiol 44:261-290. Medline

Meredith GE, Rademacher DJ (2011) MPTP mouse models of Parinson's disease: an update. J Parkinsons Dis 1:19-33. CrossRef Medline

Meredith GE, Farrell T, Kellaghan P, Tan Y, Zahm DS, Totterdell S (1999) Immunocytochemical characterization of catecholaminergic neurons in the rat striatum following dopamine-depleting lesions. Eur J Neurosci 11:3585-3596. CrossRef Medline

Millar J, Pelling CW (2001) Improved methods for construction of carbon fibre electrodes for extracellular spike recording. J Neurosci Methods 110:1-8. CrossRef Medline

Miller DW, Abercrombie ED (1999) Role of high-affinity dopamine uptake and impulse activity in the appearance of extracellular dopamine in striatum after administration of exogenous L-DOPA: studies in intact and 6-hydroxydopamine-treated rats. J Neurochem 72:1516-1522. CrossRef Medline

Mura A, Jackson D, Manley MS, Young SJ, Groves PM (1995) Aromatic L-amino acid decarboxylase immunoreactive cells in the rat striatum: a possible site for the conversion of exogenous L-DOPA to dopamine. Brain Res 704:51-60. CrossRef Medline

Mura A, Linder JC, Young SJ, Groves PM (2000) Striatal cells containing aromatic L-amino acid decarboxylase: an immunohistochemical comparison with other classes of striatal neurons. Neuroscience 98:501-511. CrossRef Medline

Ogren SO, Hall H, Kohler C, Magnusson O, Sjostrand SE (1986) The selective dopamine D2 receptor antagonist raclopride discriminates between dopamine-mediated motor functions. Psychopharmacology (Berl) 90: 287-294. 
Palfi S, Leventhal L, Chu Y, Ma SY, Emborg M, Bakay R, Déglon N, Hantraye P, Aebischer P, Kordower JH (2002) Lentivirally delivered glial cell linederived neurotrophic factor increases the number of striatal dopaminergic neurons in primate models of nigrostriatal degeneration. J Neurosci 22:4942-4954. Medline

Parent A (1986) Comparative neurobiology of the basal ganglia. New York: Wiley

Patel J, Rice ME (2006) Dopamine release in brain slices. In: Encyclopedia of sensors, Vol 6 (Grimes CA, Dickey EC, Pishko MV, eds), pp 313-334. Stevenson Ranch, CA: American Scientific.

Petroske E, Meredith GE, Callen S, Totterdell S, Lau YS (2001) Mouse model of Parkinsonism: a comparison between subacute MPTP and chronic MPTP/probenecid treatment. Neuroscience 106:589-601. CrossRef Medline

Pikaart MJ, Recillas-Targa F, Felsenfeld G (1998) Loss of transcriptional activity of a transgene is accompanied by DNA methylation and histone deacetylation and is prevented by insulators. Genes Dev 12:2852-2862. CrossRef Medline

Porritt MJ, Batchelor PE, Hughes AJ, Kalnins R, Donnan GA, Howells DW (2000) New dopaminergic neurons in Parkinson's disease striatum. Lancet 356:44-45. CrossRef Medline

Robinson DL, Wightman RM (2004) Nomifensine amplifies subsecond dopamine signals in the ventral striatum of freely-moving rats. J Neurochem 90:894-903. CrossRef Medline

Robinson DL, Heien ML, Wightman RM (2002) Frequency of dopamine concentration transients increases in dorsal and ventral striatum of male rats during introduction of conspecifics. J Neurosci 22:10477-10486. Medline

Roeper J (2013) Dissecting the diversity of midbrain dopamine neurons. Trends Neurosci 36: 336-342. CrossRef Medline

Roitman MF, Stuber GD, Phillips PE, Wightman RM, Carelli RM (2004) Dopamine operates as a subsecond modulator of food seeking. J Neurosci 24:1265-1271. Medline

San Sebastián W, Guillén J, Manrique M, Belzunegui S, Ciordia E, IzalAzcárate A, Garrido-Gil P, Vázquez-Claverie M, Luquin MR (2007) Modification of the number and phenotype of striatal dopaminergic cells by carotid body graft. Brain 130:1306-1316. CrossRef Medline

Tandé D, Höglinger G, Debeir T, Freundlieb N, Hirsch EC, François C (2006) New striatal dopamine neurons in MPTP-treated macaques result from a phenotypic shift and not neurogenesis. Brain 129:1194-1200. CrossRef Medline

Tashiro Y, Kaneko T, Sugimoto T, Nagatsu I, Kikuchi H, Mizuno N (1989)
Striatal neurons with aromatic L-amino acid decarboxylase-like immunoreactivity in the rat. Neurosci Lett 100:29-34. CrossRef Medline

Tecuapetla F, Patel JC, Xenias H, English D, Tadros I, Shah F, Berlin J, Deisseroth K, Rice ME, Tepper JM, Koos T (2010) Glutamatergic signaling by mesolimbic dopamine neurons in the nucleus accumbens. J Neurosci 30:7105-7110. CrossRef Medline

Tepper JM, Bolam JP (2004) Functional diversity and specificity of neostriatal interneurons. Curr Opin Neurobiol 14:685-692. CrossRef Medline

Tepper JM, Tecuapetla F, Koós T, Ibáñez-Sandoval O (2010) Heterogeneity and diversity of striatal GABAergic interneurons. Front Neuroanat 4:150. CrossRef Medline

Ugrumov MV (2009) Non-dopaminergic neurons partly expressing dopaminergic phenotype: distribution in the brain, development and functional significance. J Chem Neuroanat 38:241-256. CrossRef Medline

Ünal B, Ibáñez-Sandoval O, Shah F, Abercrombie ED, Tepper JM (2011) Distribution of tyrosine hydroxylase-expressing interneurons with respect to anatomical organization of the neostriatum. Front Syst Neurosci 5:41. CrossRef Medline

Ünal B, Shah F, Kothari J, Tepper JM (2015) Anatomical and electrophysiological changes in striatal tyrosine hydroxylase-expressing interneurons after nigrostriatal dopamine loss. Brain Struct Funct 220:331-449. CrossRef Medline

Weihe E, Depboylu C, Schütz B, Schäfer MK, Eiden LE (2006) Three types of tyrosine hydroxylase-positive CNS neurons distinguished by dopa decarboxylase and VMAT2 co-expression. Cell Mol Neurobiol 26:659-678. CrossRef Medline

Westin JE, Andersson M, Lundblad M, Cenci MA (2001) Persistent changes in striatal gene expression induced by long-term L-DOPA treatment in a rat model of Parkinson's disease. Eur J Neurosci 14:1171-1176. CrossRef Medline

Wiedemann DJ, Kawagoe KT, Kennedy RT, Ciolkowski EL, Wightman RM (1991) Strategies for low detection limit measurements with cyclic voltammetry. Anal Chem 63:2965-2970. CrossRef Medline

Wilson CJ (2007) GABAergic inhibition in the neostriatum. Prog Brain Res 160:91-110. CrossRef Medline

Zigmond MJ, Hastings TG, Abercrombie ED (1992) Neurochemical responses to 6-hydroxydopamine and L-dopa therapy: implications for Parkinson's disease. Ann N Y Acad Sci 648:71-86. CrossRef Medline

Zoli M, Agnati LF, Tinner B, Steinbusch HW, Fuxe K (1993) Distribution of dopamine-immunoreactive neurons and their relationships to transmitter and hypothalamic hormone-immunoreactive neuronal systems in the rat mediobasal hypothalamus. A morphometric and microdensitometric analysis. J Chem Neuroanat 6:293-310. CrossRef Medline 